\title{
Robust stabilization of delayed neural fields with partial measurement and actuation ${ }^{1}$
}

\author{
Antoine Chaillet ${ }^{\mathrm{a}, \mathrm{b}}$, Georgios Is. Detorakis ${ }^{\mathrm{a}}$, Stéphane Palfi ${ }^{\mathrm{c}}$, Suhan Senova ${ }^{\mathrm{c}}$ \\ ${ }^{\text {a } L 2 S, ~ C e n t r a l e S u p e ́ l e c ~-~ U n i v . ~ P a r i s ~ S u d-C N R S, ~ U n i v . ~ P a r i s ~ S a c l a y . ~ 3, ~ r u e ~ J o l i o t-C u r i e, ~ 91192, ~ G i f-s u r-Y v e t t e, ~ F r a n c e ~}$ \\ ${ }^{\mathrm{b}}$ Junior member of Institut Universitaire de France (IUF). \\ ${ }^{\mathrm{c}}$ Hospital H. Mondor - Service de neurochirurgie, Inserm - U955 - Eq. 14, Univ. Paris Est - Faculty of Medicine, Créteil, \\ France
}

\begin{abstract}
Neural fields are integro-differential equations describing spatiotemporal activity of neuronal populations. When considering finite propagation speed of action potentials, neural fields are affected by space-dependent delays. In this paper, we provide conditions under which such dynamics can be robustly stabilized by a proportional feedback acting only on a portion of the neuronal population and by relying on measurements of this subpopulation only. To that aim, in line with recent works, we extend the concept of input-to-state stability (ISS) to generic nonlinear delayed spatiotemporal dynamics and provide a small-gain result relying on Lyapunov-Krasovskii functionals. Exploiting the robustness properties induced by ISS, we provide conditions under which a uniform control signal can be used for the whole controlled subpopulation and we analyze the robustness of the proposed strategy to measurement and actuation delays. These theoretical findings are compared to simulation results in a model of pathological oscillations generation in Parkinson's disease.
\end{abstract}

Key words: Delayed neural fields, robust stabilization, input-to-state stability, spatiotemporal delayed systems.

\section{Introduction}

The use of spatiotemporal models to describe the activity of neuronal populations is considerably increasing. This success is due to technological advances, including electrode arrays and brain imaging, that allow recordings of unprecedented resolution in both time and space. The possibility to represent the spatiotemporal evolution of neural activity constitutes a crucial feature to deepen our understanding of cerebral functions or diseases that involve propagating waves or pattern formation, such as memory, epilepsy, or Parkinson's disease (Bressloff, 2012; Coombes et al., 2014).

Neural fields are nonlinear integro-differential equations designed to model the spatiotemporal evolution of neuronal populations. They offer a good compromise between physiological plausibility, richness of behaviors, and analytical tractability. Neural fields dynamics are derived based on statistical considerations by assuming that neural activity evolves on a continuous medium. They rely on the seminal works Wilson and Cowan (1973); Amari (1977). They have been the subject of an intense research, with a wide range of applications to neuroscience: we refer the reader to Bressloff (2012) for a detailed survey on neural fields. From an analytical point of view, several works have been devoted to the existence and estimation of equilibrium patterns, local and global stability analysis, bifurcation

Email addresses: antoine.chaillet@centralesupelec.fr (Antoine Chaillet), georgios.detorakis@lss.supelec.fr (Georgios Is. Detorakis), stephane.palfi@aphp.fr (Stéphane Palfi), yannsuhansenova@gmail.com (Suhan Senova).

1 This work is supported by a public grant overseen by the French National Research Agency (ANR) as part of the "Investissement d'Avenir" program, through the "iCODE Institute project" funded by the IDEX Paris-Saclay, ANR-11-IDEX-0003-02, and by the ANR JCJC project SynchNeuro. 
analysis, and existence of periodic orbits: see e.g. Pinto and Ermentrout (2001); Laing et al. (2002); Faugeras et al. (2009); Veltz and Faugeras (2010).

A key source of complexity, and subsequent richness of producible dynamical behaviors, lies in the delays induced by the non-instantaneous communication between neurons. From a physiological perspective, these delays are needed to model the finite speed of signals along axons. They typically depend on the physical distance between the considered neurons. Delayed neural fields have been the subject of several mathematical studies, including Atay and Hutt (2004, 2006); Veltz and Faugeras (2011); beim Graben and Hutt (2014).

A central question in the analytical study of neural fields stands in the stability of stationary solutions. This question is crucial for the understanding of the resulting brain function as modeled by the equilibrium pattern. In some applications, it is also motivated by the need for non-oscillating responses. In the case of Parkinson's disease, which is the original motivation for the present work, such oscillations in specific frequency bands are correlated with motor symptoms. The mechanisms for this pathological oscillations onset are still a matter of debate, but are believed to be linked to the combination of propagation delays and exaggerated synaptic weights between neuronal populations (Plenz and Kital, 1999; Nevado-Holgado et al., 2010; Pasillas-Lépine, 2013). Following this hypothesis, a natural way to improve motor performance would be to attenuate these pathological oscillations, by relying on electrical (Benabid et al., 1991) or optical (Gradinaru et al., 2009) stimulation. This was investigated in Haidar et al. (2016) by relying on a model of the averaged activity, and then extended in Detorakis et al. (2015) to spatiotemporal dynamics.

Despite numerous theoretical developments on neural fields, with or without delays, the question of their stabilization by a control signal had not yet been addressed. The purpose of this note is to analyze the stability and robustness induced by proportional feedback control. In order to cope with implementation constraints, we consider the case when the stimulation signal impacts directly only a portion of the neuronal population, which we refer to as the "controlled population". We also impose that the feedback relies only on measurements on this controlled population in order to limit the number of required sensing electrodes. Our main result states that global robust stabilization of delayed neural fields can always be achieved provided that the internal synaptic strength of the "uncontrolled" population is lower than the inverse of the Lipschitz constant of the activation function. This result in turn corrects a flaw in the (incomplete) proof provided in Detorakis et al. (2015). We also address two constraints of practical relevance, which were addressed only through simulations in Detorakis et al. (2015). The first one is the limited number of available stimulation points: we show that stabilization can still be achieved with a unique control signal provided that the synaptic weights are homogeneous enough. The second one is the unavoidable feedback delays needed to acquire and estimate the neural activity: we show that the considered proportional scheme is robust to such delays but that, not surprisingly, this robustness may decrease as the feedback gain increases.

In order to establish these results, we rely on a spatiotemporal extension of input-to-state stability (ISS, Sontag $(1989,2008))$. This extension imposes that the state's $L_{2}$-norm over the spatial domain be bounded by a vanishing function of the spatial $L_{2}$-norm of initial conditions plus a term "proportional" to the spatial $L_{2}$-norm of exogenous inputs. A further extension is required in order to allow for delayed dynamics. After having extended the classical Lyapunov sufficient condition for ISS (Sontag, 1989), we present a small-gain theorem applicable to such class of systems (Section 3). This extension is in line with recent works addressing ISS for infinite-dimensional systems (Dashkovskiy and Mironchenko, 2013; Karafyllis and Jiang, 2007; Mironchenko and Ito, 2016; Prieur and Mazenc, 2012), including retarded functional differential equations (Pepe and Jiang, 2006; Karafyllis et al., 2008; Mazenc et al., 2008; Teel, 1998). Focusing on delayed neural fields under partial proportional feedback, we then show that the uncontrolled population is ISS with respect to the state of the controlled population and possible exogenous signals, provided that the spatial $L_{2}$-norm of its internal synaptic weights is below a certain bound. On the other hand, we show that the controlled population can always be made ISS by picking a sufficiently large feedback gain. ISS of the overall closed-loop system is then established based on small-gain arguments. Both stabilization by a single stimulation signal and robustness to feedback delays are addressed by exploiting the robustness induced by the ISS property (Section 4). Simulations are then presented in Section 5 to confirm our theoretical expectancies and to evaluate the performance of the considered control laws (proportional, uniform, and involving feedback delays).

Notation. Given $x=\left(x_{1}, \ldots, x_{n}\right) \in \mathbb{R}^{n},|x|$ denotes its Euclidean norm: $|x|:=\sqrt{x_{1}^{2}+\ldots+x_{n}^{2}}$. Given two sets $\Omega_{1}$ and $\Omega_{2}, C\left(\Omega_{1}, \Omega_{2}\right)$ (resp. $C^{1}\left(\Omega_{1}, \Omega_{2}\right)$ ) denotes the set of all continuous (resp. continuously differentiable) functions from $\Omega_{1}$ to $\Omega_{2}$. $L_{2}\left(\Omega_{1}, \Omega_{2}\right)$ denotes the set of all square integrable functions from $\Omega_{1}$ to $\Omega_{2}$, meaning all functions $f: \Omega_{1} \rightarrow \Omega_{2}$ such that $\int_{\Omega_{1}}|f(s)|^{2} d s<\infty$. Given a set $\Omega \subset \mathbb{R}^{q}, \#_{\Omega}$ denotes its Lebesgue measure. Given $f: \Omega \rightarrow \mathbb{R}^{n}$, with $\Omega=\Omega_{1} \times \ldots \Omega_{q}$ where $\Omega_{i} \subset \mathbb{R}$ for each $i \in\{1, \ldots, q\}$, we compactly write $\int_{\Omega} f(r) d r$ to denote the multiple integral $\int_{\Omega_{1}} \ldots \int_{\Omega_{q}} f(r) d r_{q} \ldots d r_{1}$, with $r=:\left(r_{1}, \ldots, r_{q}\right)^{T}$. We define $\mathcal{F}^{n}:=L_{2}\left(\Omega, \mathbb{R}^{n}\right)$ and $\mathcal{C}^{n}:=C\left([-\bar{d} ; 0], \mathcal{F}^{n}\right)$ for 
some constant $\bar{d}>0 . \mathcal{F}^{n}$ is a Banach space for the $L_{2^{-n o r m}}\|\cdot\| \mathcal{F}^{n}$ defined as $\|x\|_{\mathcal{F}^{n}}:=\sqrt{\int_{\Omega}|x(s)|^{2} d s}$ for each $x \in \mathcal{F}^{n}$. Similarly, $\mathcal{C}^{n}$ is a Banach space for the norm $\|\cdot\|_{\mathcal{C}^{n}}$ defined as $\|x\|_{\mathcal{C}^{n}}:=\sup _{t \in[-\bar{d} ; 0]}\|x(t)\|_{\mathcal{F}^{n}}$ for all $x \in \mathcal{C}^{n}$. We also denote by $\mathcal{U}^{n}$ the set of all measurable locally bounded functions from $\mathbb{R}_{\geq 0}$ to $\mathcal{F}^{n}$. When the context is sufficiently clear, we will simply refer to $\mathcal{F}^{n}, \mathcal{C}^{n}$ and $\mathcal{U}^{n}$ as $\mathcal{F}, \mathcal{C}$ and $\mathcal{U}$ respectively. Given $x \in \mathcal{C}$ and $t \in[-\bar{d} ; 0]$, we indicate by $[x(t)](r)$ the value taken by the function $x(t) \in \mathcal{F}$ at position $r \in \Omega$. A function $\alpha: \mathbb{R}_{\geq 0} \rightarrow \mathbb{R}_{\geq 0}$ is said to be of class $\mathcal{K}$ if it is continuous, zero at zero and increasing. It is said to be of class $\mathcal{K}_{\infty}$ if it satisfies additionally $\lim _{s \rightarrow+\infty} \alpha(s)=+\infty$. A function $\beta: \mathbb{R}_{\geq 0} \times \mathbb{R}_{\geq 0} \rightarrow \mathbb{R}_{\geq 0}$ is said to be of class $\mathcal{K} \mathcal{L}$ if $s \mapsto \beta(s, t)$ is of class $\mathcal{K}$ for any fixed $t \in \mathbb{R}_{\geq 0}$ and, for any fixed $s \in \mathbb{R}_{\geq 0}, t \mapsto \beta(s, \bar{t})$ is continuous and non-increasing and tends to zero as its argument tends to $+\infty$.

\section{Delayed neural fields}

We start by introducing the spatiotemporal model under concern. Delayed neural fields are integro-differential equations of the form:

$$
\tau_{i}(r) \frac{\partial z_{i}}{\partial t}(r, t)=-z_{i}(r, t)+\mathcal{S}_{i}\left(\sum_{j=1}^{n} \int_{\Omega} w_{i j}\left(r, r^{\prime}\right) z_{j}\left(r^{\prime}, t-d_{j}\left(r, r^{\prime}\right)\right) d r^{\prime}+I_{i}^{\mathrm{ext}}(r, t)\right)
$$

for $i \in\{1, \ldots, n\} . \Omega$ denotes a set of $\mathbb{R}^{q}, q \in\{1,2,3\}$, representing the physical support of the populations; throughout this paper, we will assume that $\Omega$ is compact. $r, r^{\prime} \in \Omega$ are the space variables, whereas $t \in \mathbb{R}_{\geq 0}$ is the time variable. $z_{i}(r, t) \in \mathbb{R}$ represents the neuronal activity of population $i$, at position $r \in \Omega$, and at time $t \in \mathbb{R}_{\geq 0} \cdot \tau_{i}(r)>0$ is the time decay constant of the activity of population $i$ at position $r \in \Omega$. The kernel $w_{i j}: \Omega \times \Omega \rightarrow \mathbb{R}$ is a bounded function describing the synaptic strength between location $r^{\prime}$ in population $j$ and location $r$ in population $i$. $I_{i}^{\text {ext }}: \Omega \times \mathbb{R}_{\geq 0} \rightarrow \mathbb{R}$ is a bounded function describing the external input of population $i$, arising either from the influence of exogenous cerebral structures or from an artificial stimulation device. The function $d_{j}: \Omega \times \Omega \rightarrow[0 ; \bar{d}]$, $\bar{d} \geq 0$, is a continuous function representing the axonal, dendritic and synaptic delays between a pre-synaptic neuron at position $r^{\prime}$ in population $j$ and a post-synaptic neuron at position $r$. $\mathcal{S}_{i}: \mathbb{R} \rightarrow \mathbb{R}$ is a nondecreasing globally Lipschitz function, known as the activation function of the neural population $i$.

The compactness assumption on $\Omega$ is realistic as neuronal populations are typically circumscribed to a limited physical domain. Although $\tau_{i}$ is assumed to be uniform in most existing works on neural fields, we allow it to depend on the position $r$ for the sake of generality. Similarly, in most neuroscience works, the synaptic kernels $w_{i j}$ are assumed to depend only on the distance between $r$ and $r^{\prime}$ (i.e. $\left.w_{i j}\left(r, r^{\prime}\right)=w_{i j}\left(\left|r-r^{\prime}\right|\right)\right)$. Typical shapes include Gaussian functions or "Mexican hats". Here we allow for more generic kernels, as they may encompass non-homogeneous synaptic weights distributions. The delay distributions $d_{j}$ are also allowed to depend on positions $r$ and $r^{\prime}$ in an inhomogeneous manner, although a typical model is $d_{j}\left(r, r^{\prime}\right)=\left|r-r^{\prime}\right| / c_{j}$, where $c_{j}$ is the axonal propagation speed of neurons in population $j$. These delays are neglected in several analytical works on neural fields (see e.g. Pinto and Ermentrout (2001); Laing et al. (2002); Faugeras et al. (2009); Veltz and Faugeras (2010)), but they are crucial in the present study as they are likely to play a key role in the generation of oscillations linked to Parskinson's disease (Nevado-Holgado et al., 2010; Pasillas-Lépine, 2013).

\subsection{Controlled and non-controlled populations}

Let us consider the impact of a stimulation signal on the populations network modeled by (1) with only two interacting subpopulations $(n=2)$. We assume that this stimulation signal acts directly on a given subpopulation only. In the case of optogenetics, this subpopulation corresponds to the set of neurons that express the gene that codes for photoactivatable proteins and that receive sufficient illumination from the stimulation device. Without loss of generality, we consider that this "controlled" population corresponds to the index $i=1$. The dynamics of this population then reads

$$
\tau_{1}(r) \frac{\partial z_{1}}{\partial t}(r, t)=-z_{1}(r, t)+\mathcal{S}_{1}\left(\sum_{j=1}^{2} \int_{\Omega} w_{1 j}\left(r, r^{\prime}\right) z_{j}\left(r^{\prime}, t-d_{j}\left(r, r^{\prime}\right)\right) d r^{\prime}+I_{1}+\alpha(r) u\right)
$$


where some variables have been omitted for the sake of writing compactness. $I_{1}: \Omega \times \mathbb{R}_{\geq 0} \rightarrow \mathbb{R}$ is an exogenous input (resulting from the influence of unmodelled cerebral structures) and $u: \Omega \times \mathbb{R}_{\geq 0} \rightarrow \mathbb{R}$ is the stimulation signal that we use for control. $\alpha: \Omega \rightarrow \mathbb{R}_{>0}$ is a gain distribution accounting for the strength of the stimulation influence. It typically decreases as a function of the distance to the stimulation device, due to light (Jacques, 2013) and electrical (Butson and McIntyre, 2005) absorption in brain tissues. The fact that $\alpha(r)>0$ for each $r \in \Omega$ means that each neuron of the controlled population receives a sufficient stimulation, so that its activity can be modulated by the control signal $u$.

The other population, $i=2$, is not directly affected by the stimulation signal: the impact of the stimulation signal on this population may only result from its synaptic connection with population 1 . We refer to this population as "uncontrolled". Its dynamics reads:

$$
\tau_{2}(r) \frac{\partial z_{2}}{\partial t}(r, t)=-z_{2}(r, t)+\mathcal{S}_{2}\left(\sum_{j=1}^{2} \int_{\Omega} w_{2 j}\left(r, r^{\prime}\right) z_{j}\left(r^{\prime}, t-d_{j}\left(r, r^{\prime}\right)\right) d r^{\prime}+I_{2}(r, t)\right)
$$

where $I_{2}: \Omega \times \mathbb{R}_{\geq 0} \rightarrow \mathbb{R}$ gathers influences from other brain structures.

\subsection{Problem statement}

The main question addressed in this paper is the following: is it possible to design a feedback control law $u$, relying only on measurements on the controlled population, such that the coupled neural fields (2)-(3) admit an equilibrium pattern to which all solutions converge?

Although it may be of interest to other neuroscience problems, this question was originally motivated by the development of innovative brain stimulation strategies to compensate motor symptoms in Parkinson's disease. We refer the reader to Detorakis et al. (2015) for a more detailed discussion on the employed strategy and its practical implementability. In short, some parkinsonian motor symptoms are correlated to excessive oscillations, in the frequency band $13-30 \mathrm{~Hz}$, in a deep brain region known as the subthalamic nucleus (STN). The mechanisms underlying the generation of such pathological oscillations are still debated. One hypothesis, defended in e.g. Plenz and Kital (1999); Nevado-Holgado et al. (2010); Pasillas-Lépine (2013), is that these oscillations may result from a too strong synaptic interaction between STN and a narrow structure called external globus pallidus (GPe): these too strong synaptic weights, combined with the inherent propagation delays between the two populations, would tend to induce oscillating responses. It is thus of interest to study the possibility to attenuate these pathological oscillations using closed-loop techniques. As reported in the survey paper (Carron et al., 2013), several attempts have been made in that direction. These include adaptive and on-demand stimulation (Rosin et al., 2011; Graupe et al., 2010; Santaniello et al., 2011; Little et al., 2016; Marceglia et al., 2007), delayed and multi-site stimulation (Lysyansky et al., 2011; Batista et al., 2010; Pfister and Tass, 2010; Tass et al., 2012), optimal control strategies (Feng et al., 2007), and activity regulation (Haidar et al., 2016; Wagenaar et al., 2005; Grant and Lowery, 2013).

Beyond the goal of stabilizing the coupled neural fields (2)-(3), robustness questions also need to be addressed. A first reason for that stands in the unavoidable uncertainty and variability induced by in vivo experiments. Another reason is more related to technological considerations, including the time needed to acquire and process the STN activity in real time, and the limited stimulation sources available in practice.

\subsection{Proposed feedback laws}

The simplest closed-loop strategy one could think of in order to stabilize (2)-(3) is proportional control:

$$
u(r, t)=-k\left(z_{1}(r, t)-z_{r e f}(r)\right),
$$

where $k$ is a positive control gain and $z_{\text {ref }}: \Omega \rightarrow \mathbb{R}$ represents a prescribed rate for the $z_{1}$ population. At each point of the controlled population, it generates a stimulation proportional to the difference between the measured local activity and the prescribed one. A similar strategy was adopted in Haidar et al. (2016) on an averaged model (meaning neglecting spatial dependency) by relying on linearization techniques. 
This control scheme is appealing due to the computational simplicity of its implementation, as it essentially involves no other computations than the ones needed to estimate the real-time activity of the controlled population. Nevertheless, it requires the measurement of the activity at each single point of the controlled population. Since the activity is typically acquired through implanted electrodes or electrode arrays, the spatial resolution of measurements is limited in practice. Moreover, the control signal (4) depends on $r$ : it thus requires to deliver a stimulation that may differ from one position to another. Despite progresses in holographic techniques (Reutsky-Gefen et al., 2013), this may be hard to achieve in practice. A more realistic strategy would be to rely on the averaged activity of the controlled population:

$$
u(t)=-k \int_{\Omega} \alpha^{\prime}(r)\left(z_{1}(r, t)-z_{r e f}(r)\right) d r
$$

where $\alpha^{\prime}: \Omega \rightarrow \mathbb{R}_{>0}$ is a bounded function accounting for the possible inhomogeneity and absorption of the medium. Contrarily to (4), this control signal can be delivered through a unique stimulation device since it is the same for all neurons of the controlled population (although its influence on the population will locally be modulated by the distribution $\alpha$ ). Moreover, it does not necessarily require to record the local activity in each point of the controlled population, but only a spatial average of its activity (weighted by the function $\alpha^{\prime}$ ).

\section{ISS for spatiotemporal delayed dynamics}

The approach we follow in order to study the stability of the delayed neural fields (2)-(3) under the closed-loop stimulation (4) consists in studying separately the uncontrolled and controlled populations (Sections 4.2 and 4.3 respectively) by seeing the input arising from the other population as an exogenous input. By relying on small-gain arguments, we conclude that the overall system is robustly asymptotically stable (Section 4.4). Then, in Section 4.5, we establish the stabilizing properties of the uniform control law (5) by seeing it as a "perturbation" of the nominal signal (4). This robustness analysis allows in turn to study the impact of a control delay, due to the unavoidable time necessary to acquire and process the activity of the controlled population (Section 4.6).

All these developments require to adapt the concept of input-to-state stability (Sontag, 1989, 2008) and the corresponding ISS small-gain theorem (Jiang et al., 1996) to delayed neural fields. We develop this in a wider context, by considering delayed dynamical systems whose state is a function of space variables.

\subsection{Considered class of systems}

The considered delayed neural fields are infinite dimensional not only because they involve delays, but also because of their spatiotemporal nature. As observed in e.g. Faye and Faugeras (2010), such systems can actually be casted into the more general setup of a delayed state-space representation whose state is a function of the space variable $r$. More precisely, (2)-(3) in closed loop with either (4) or (5) can be seen as a particular case of the more general dynamics $^{2}$

$$
\dot{x}(t)=f\left(x_{t}, p(t)\right)
$$

where $f: \mathcal{C}^{n} \times \mathcal{F}^{m} \rightarrow \mathcal{F}^{n}$ is Lipschitz on each bounded set of $\mathcal{C}^{n} \times \mathcal{F}^{m}$ and $p \in \mathcal{U}^{m} . x(t) \in \mathcal{F}^{n}$ represents the state of this system: at each time instant $t$, it is a function of the space variable rather than a single point of $\mathbb{R}^{n}$. $x_{t} \in \mathcal{C}^{n}$ represents the history of this function over the latest time interval of length $\bar{d}$; in other words, for each fixed $\theta \in[-\bar{d} ; 0], x_{t}(\theta):=x(t+\theta)$ is a function (of the space variable) belonging to $\mathcal{F}^{n}$. Given any $t \geq 0, \dot{x}(t)$ is the function defined as $r \mapsto \frac{\partial}{\partial t}([x(t)](r))$ everywhere it exists. From now on, unless explicitly stated, $\overline{\mathcal{C}}^{n}, \mathcal{F}^{n}$, and $\mathcal{U}^{m}$ will be simply written as $\mathcal{C}, \mathcal{F}$ and $\mathcal{U}$.

\subsection{Input-to-state stability}

A natural extension of input-to-state stability to the considered class of systems is as follows.

\footnotetext{
$\overline{2}$ Due to the fact that $p \in \mathcal{U}^{m},(6)$ holds for almost all $t \geq 0$.
} 
Definition 1 (ISS) The system (6) is input-to-state stable (ISS) if there exist $\beta \in \mathcal{K} \mathcal{L}$ and $\nu \in \mathcal{K}_{\infty}$ such that, for any initial condition $x_{0} \in \mathcal{C}$ and any input $p \in \mathcal{U}$, the system (6) admits a unique solution defined over $[-\bar{d} ;+\infty)$ and it satisfies, for all $t \in \mathbb{R}_{\geq 0}$,

$$
\|x(t)\|_{\mathcal{F}} \leq \beta\left(\left\|x_{0}\right\|_{\mathcal{C}}, t\right)+\nu\left(\operatorname{ess}_{\sup } \operatorname{se[0;t]}_{\left.\|p(\tau)\|_{\mathcal{F}}\right)}\right.
$$

$\nu$ is then called an ISS gain for (6).

This definition of ISS is a particular case of the very general input-to-output stability notion introduced in Karafyllis and Jiang (2007), which applies to a wide class of systems, satisfying a weak semigroup property and encompassing in particular retarded functional differential equations, difference equations, and hybrid dynamics.

Definition 1 is also consistent with the one used for delayed systems in e.g. Pepe and Jiang (2006); Mazenc et al. (2008). The main difference stands in the fact that, in those papers, no spatial evolution is considered. As a consequence, the left hand side of the above estimate involves the $L_{2}$-norm of the solution over the spatial domain (i.e. the $\mathcal{F}$-norm), rather than a Euclidean norm of the state like in the original finite-dimensional delay-free case (Sontag, 1989, 2008). For the same reason, the magnitude of the initial condition is here estimated through its $\mathcal{C}$-norm, meaning the maximum value of its spatial $L_{2}$-norm over its interval of definition $[-\bar{d} ; 0]$.

The above property is also similar to the ISS property for infinite dimensional systems in Banach spaces introduced in Dashkovskiy and Mironchenko (2013) and extensively studied in Mironchenko (2012). The main difference stands in the fact that the state estimate in Definition 1 involves the $\mathcal{F}$-norm of the state as a function of the $\mathcal{C}$-norm of the initial condition, whereas the ISS notion used in Dashkovskiy and Mironchenko (2013) would involve the $\mathcal{C}$-norm on both sides of this estimate. It was shown in (Mironchenko, 2012, Proposition 1.4.2) that these estimates are qualitatively equivalent. Nevertheless, we have preferred this two-norm estimate as the corresponding LyapunovKrasovskii results presented below will rely on each of these two norms.

Similarly to the original ISS property for finite-dimensional systems and to its delayed extension, the above property ensures the asymptotic gain property:

$$
\limsup _{t \rightarrow+\infty}\|x(t)\|_{\mathcal{F}} \leq \nu\left(\limsup _{t \rightarrow+\infty}\|p(t)\|_{\mathcal{F}}\right) .
$$

This means that the steady-state error in the state, measured through its $\mathcal{F}$-norm, results solely from the asymptotic value of the applied input's $\mathcal{F}$-norm and is "proportional" to it, up to the scaling factor $\nu$. This in turn shows that the original "converging input-converging state" and "bounded input-bounded state" properties (Sontag, 2008) are also preserved. Furthermore, ISS ensures asymptotic stability in the absence of input, namely:

$$
p \equiv 0 \quad \Rightarrow \quad\|x(t)\|_{\mathcal{F}} \leq \beta\left(\left\|x_{0}\right\|_{\mathcal{C}}, t\right), \quad \forall t \in \mathbb{R}_{\geq 0}
$$

In particular, when no exogenous input applies, the $\mathcal{F}$-norm of the solution asymptotically converges to zero (attractiveness), and its amplitude is arbitrarily small at all times provided that $\left\|x_{0}\right\|_{\mathcal{C}}$ is small enough (stability).

\subsection{Lyapunov-Krasovskii condition for ISS}

We now present an extension to the class of systems (6) of the Lyapunov-Krasovskii condition for ISS. In line with Faye and Faugeras (2010), it relies on the upper right Dini derivative of a functional $V \in C\left(\mathcal{C}, \mathbb{R}_{\geq 0}\right)$ along the solutions of $(6)$, as defined by $\dot{V}^{(6)}:=\limsup _{h \rightarrow 0^{+}} \frac{V\left(x_{t+h}\right)-V\left(x_{t}\right)}{h}$, where $x(\cdot)$ denotes any solution of $(6)$.

Theorem 1 (Sufficient condition for ISS) Let $\underline{\alpha}, \bar{\alpha}, \alpha, \gamma \in \mathcal{K}_{\infty}$ and let $V \in C\left(\mathcal{C}, \mathbb{R}_{\geq 0}\right)$ be locally Lipschitz. Assume that, given any initial condition $x_{0} \in \mathcal{C}$ whose time derivative is continuous and any input $p \in \mathcal{U}$, the system (6) admits a unique solution defined over $[-\bar{d} ;+\infty)$ and satisfying, for almost all $t \in \mathbb{R}_{\geq 0}$,

$$
\begin{gathered}
\underline{\alpha}\left(\|x(t)\|_{\mathcal{F}}\right) \leq V\left(x_{t}\right) \leq \bar{\alpha}\left(\left\|x_{t}\right\|_{\mathcal{C}}\right) \\
V\left(x_{t}\right) \geq \gamma\left(\|p(t)\|_{\mathcal{F}}\right) \Rightarrow \dot{V}^{(6)} \leq-\alpha\left(V\left(x_{t}\right)\right) .
\end{gathered}
$$

Then the system (6) is ISS with gain $\underline{\alpha}^{-1} \circ \gamma$. 
This result is a spatiotemporal extension of (Pepe and Jiang, 2006, Theorem 3.1). Its proof, provided in Section 8.1, follows along the same lines as the ISS Lyapunov sufficient condition for finite-dimensional systems originally proposed in Sontag (1989). A similar sufficient condition for ISS of infinite-dimensional systems was proposed in Dashkovskiy and Mironchenko (2013), and further extended to integral ISS in Mironchenko and Ito (2016). The main difference with Theorem 1 stands in the fact that, in those works, the Lyapunov functional $V$ is assumed to be upper and lower-bounded by a function of $\left\|x_{t}\right\|_{\mathcal{C}}$ (whereas (9) requires only a lower-bound involving $\|x(t)\|_{\mathcal{F}}$ ). Such a lower bound turns out to be handier for the analysis of delayed neural fields, as will be illustrated in Section 4. The fact that (9)-(10) are required to hold only for almost all $t \in \mathbb{R}_{\geq 0}$ results from the absolute continuity of $t \mapsto V\left(x_{t}\right)$. This results from the following observation, which can be derived similarly to (Pepe, 2007, Lemma 4) by replacing the Euclidean norm by the $\mathcal{F}$-norm.

Lemma 1 For any $x_{0} \in \mathcal{C}$ whose time-derivative is continuous and for any essentially bounded $p \in \mathcal{U}$, if $(6)$ is forward complete then its solution $x(\cdot)$ is such that $t \mapsto x_{t}$ is absolutely continuous on $\mathbb{R}_{\geq 0}$ with respect to the $\mathcal{C}$-norm.

Lemma 1 considers only initial conditions that are continuously differentiable in time. As observed in Pepe (2007) in a non-spatiotemporal context, this induces no loss of generality, which explains why (9)-(10) need to be tested only for continuously differentiable initial states. We formally state this as the following lemma, whose proof follows readily from that of (Pepe, 2007, Proposition 3) after observing that $C^{1}([-\bar{d} ; 0], \mathcal{F})$ is dense in $\mathcal{C}$.

Lemma 2 The system (6) is ISS if and only if it is ISS for all initial conditions $x_{0} \in \mathcal{C}$ with continuous time derivative.

\subsection{ISS small-gain theorem}

We next address the stability of a feedback interconnection by extending the ISS small-gain theorem to systems of the form (6). More precisely, we consider feedback systems of the form

$$
\begin{aligned}
& \dot{x}_{1}(t)=f_{1}\left(x_{1 t}, x_{2 t}, p_{1}(t)\right) \\
& \dot{x}_{2}(t)=f_{2}\left(x_{2 t}, x_{1 t}, p_{2}(t)\right),
\end{aligned}
$$

where $f_{1}: \mathcal{C}^{n_{1}} \times \mathcal{C}^{n_{2}} \times \mathcal{F}^{m_{1}} \rightarrow \mathcal{F}^{n_{1}}, f_{2}: \mathcal{C}^{n_{2}} \times \mathcal{C}^{n_{1}} \times \mathcal{F}^{m_{2}} \rightarrow \mathcal{F}^{n_{2}}, p_{1} \in \mathcal{U}^{m_{1}}$ and $p_{2} \in \mathcal{U}^{m_{2}}$. For these systems, we have the following result.

Theorem 2 (ISS small gain) For each $i \in\{1,2\}$, let $\underline{\alpha}_{i}, \bar{\alpha}_{i}, \alpha_{i}, \gamma_{i}, \chi_{i} \in \mathcal{K}_{\infty}$ and $V_{i} \in C\left(\mathcal{C}^{n_{i}}, \mathbb{R}_{\geq 0}\right)$ be locally Lipschitz. Assume that, given any $x_{i 0} \in \mathcal{C}^{n_{i}}$ with continuous time derivative and any $p_{i} \in \mathcal{U}^{m_{i}}$, the system (11) admits a unique solution defined over $[-\bar{d} ;+\infty)$ and satisfying, for almost all $t \in \mathbb{R}_{\geq 0}$,

$$
\begin{gathered}
\underline{\alpha}_{i}\left(\left\|x_{i}(t)\right\|_{\mathcal{F}}\right) \leq V_{i}\left(x_{i t}\right) \leq \bar{\alpha}_{i}\left(\left\|x_{i t}\right\|_{\mathcal{C}}\right) \\
V_{1} \geq \max \left\{\chi_{1}\left(V_{2}\right), \gamma_{1}\left(\left\|p_{1}(t)\right\|_{\mathcal{F}}\right)\right\} \Rightarrow \dot{V}_{1}^{(11 \mathrm{a})} \leq-\alpha_{1}\left(V_{1}\right) \\
V_{2} \geq \max \left\{\chi_{2}\left(V_{1}\right), \gamma_{2}\left(\left\|p_{2}(t)\right\|_{\mathcal{F}}\right)\right\} \Rightarrow \dot{V}_{2}^{(11 \mathrm{~b})} \leq-\alpha_{2}\left(V_{2}\right) .
\end{gathered}
$$

Then, under the small-gain condition $\chi_{1} \circ \chi_{2}(s)<s$ for all $s>0$, the feedback interconnection (11) is ISS.

This result, proved in Section 8.2, is a natural extension of the main result in Pepe and Jiang (2006) to spatiotemporal dynamics, which was already a generalization to delayed dynamics of the ISS small-gain theorem provided in Jiang et al. (1996). It shares strong similarities with the small-gain result in Dashkovskiy and Mironchenko (2013), which goes beyond Theorem 2 by allowing for the interconnection of more than two subsystems. As stressed before, the main difference with that work stands in the fact that the Lyapunov function associated to each subsystem is here required to be lower-bounded by a function of $\|x(t)\|_{\mathcal{F}}$ only (rather than $\left\|x_{t}\right\|_{\mathcal{C}}$ ). The same obstruction holds for (Karafyllis and Jiang, 2011, Corollary 4.5), which relies on Lyapunov-Krasovskii functionals depending only on $\|x\|_{\mathcal{C}}$. A very general small-gain theorem for systems defined in Banach spaces was also provided in Karafyllis and Jiang (2007). Nonetheless, the small-gain condition presented there relies on nonlinear gains involved in the estimate of the evolution of the Lyapunov functionals $V_{i}$ rather than those involved in their dissipation inequalities. Estimating 
these nonlinear gains would thus require to first integrate the dissipation inequalities assumed in the statement of Theorem 2.

In some situations, the positive terms appearing in the dissipation inequalities cannot be easily expressed in terms of the Lyapunov functional of the other subsystem. The following result, proved in Section 8.3, can help tackling this situation.

Corollary 1 For each $i \in\{1,2\}$, let $\underline{\alpha}_{i}, \bar{\alpha}_{i}, \alpha_{i}, \gamma_{i}, \chi_{i} \in \mathcal{K}_{\infty}, V_{i}, W_{i} \in C\left(\mathcal{C}^{n_{i}}, \mathbb{R}_{>0}\right)$ be locally Lipschitz, and $k_{12}, k_{21} \geq$ 0 . Assume that, given any initial condition $x_{i 0} \in \mathcal{C}^{n_{i}}$ with continuous time derivative and any input $p_{i} \in \mathcal{U}^{m_{i}}$, the system (11) admits a unique solution defined over $[-\bar{d} ;+\infty)$ and satisfying, for almost all $t \in \mathbb{R}_{\geq 0}$, both (12) and

$$
\begin{aligned}
& \dot{V}_{1}^{(11 \mathrm{a})} \leq-\alpha_{1}\left(V_{1}\right)-W_{1}+k_{12} W_{2}+\gamma_{1}\left(\left\|p_{1}(t)\right\|_{\mathcal{F}}\right) \\
& \dot{V}_{2}^{(11 \mathrm{~b})} \leq-\alpha_{2}\left(V_{2}\right)-W_{2}+k_{21} W_{1}+\gamma_{2}\left(\left\|p_{2}(t)\right\|_{\mathcal{F}}\right)
\end{aligned}
$$

Then, under the condition $k_{12} k_{21} \leq 1$, the feedback interconnection (11) is ISS.

\section{ISS stabilization of delayed neural fields}

We now rely on the above framework to address the ISS stabilization of delayed neural fields with limited actuation and measurement.

\subsection{Closed-loop system}

The dynamics of the controlled population (2) and the uncontrolled populations (3) under the influence of the closed-loop stimulation signal (4) reads

$$
\begin{aligned}
& \tau_{1}(r) \frac{\partial z_{1}}{\partial t}(r, t)=-z_{1}(r, t)+\mathcal{S}_{1}\left(\sum_{j=1}^{2} \int_{\Omega} w_{1 j}\left(r, r^{\prime}\right) z_{j}\left(r^{\prime}, t-d_{j}\left(r, r^{\prime}\right)\right) d r^{\prime}-k \alpha(r)\left(z_{1}-z_{r e f}\right)+I_{1}(r, t)\right) \\
& \tau_{2}(r) \frac{\partial z_{2}}{\partial t}(r, t)=-z_{2}(r, t)+\mathcal{S}_{2}\left(\sum_{j=1}^{2} \int_{\Omega} w_{2 j}\left(r, r^{\prime}\right) z_{j}\left(r^{\prime}, t-d_{j}\left(r, r^{\prime}\right)\right) d r^{\prime}+I_{2}(r, t)\right),
\end{aligned}
$$

where, for notation compactness, we omitted the arguments of some functions. Given any constant inputs $I_{1}(r, t)=$ $I_{1}^{*}(r)$ and $I_{2}(r, t)=I_{2}^{*}(r)$, with $I_{1}^{*}, I_{2}^{*} \in \mathcal{F}$, let $z_{1}^{*}, z_{2}^{*} \in \mathcal{F}$ denote any equilibrium pattern of the neural fields model (13). The existence of such equilibria was ensured in (Faugeras et al., 2009) in the absence of feedback $(k=0)$ based on Schaefer's fixed point theorem. In particular, the neural fields (2)-(3) under uniform feedback (5) are guaranteed to admit an equilibrium. The methodology does not readily extend to (13) due to the non-compactness of the feedback term. For now on, we simply assume that such an equilibrium exists. Note that, for each $i \in\{1,2\}, z_{i}^{*}(r)$ is in the image of $\mathcal{S}_{i}$ for all $r \in \Omega$. For each $t \in \mathbb{R}_{\geq 0}$, let $x_{1}(t), x_{2}(t) \in \mathcal{F}$ be defined as $\left[x_{1}(t)\right](r):=z_{1}(r, t)-z_{1}^{*}(r)$ and $\left[x_{2}(t)\right](r):=z_{2}(r, t)-z_{2}^{*}(r)$ for all $r \in \Omega$. Similarly, let $p_{1}(t), p_{2}(t) \in \mathcal{F}$ be defined as $\left[p_{1}(t)\right](r):=I_{1}(r, t)-I_{1}^{*}(r)$ and $\left[p_{2}(t)\right](r):=I_{2}(r, t)-I_{2}^{*}(r)$. Then $(13)$ can be written as

$$
\begin{aligned}
& \tau_{1}(r)\left[\dot{x}_{1}(t)\right](r)=-\left[x_{1}(t)\right](r)+S_{1}\left(r,-k \alpha(r)\left[x_{1}(t)\right](r)+\sum_{j=1}^{2} \int_{\Omega} w_{1 j}\left(r, r^{\prime}\right)\left[x_{j}\left(t-d_{j}\right)\right]\left(r^{\prime}\right) d r^{\prime}+\left[p_{1}(t)\right](r)\right) \\
& \tau_{2}(r)\left[\dot{x}_{2}(t)\right](r)=-\left[x_{2}(t)\right](r)+S_{2}\left(r, \sum_{j=1}^{2} \int_{\Omega} w_{2 j}\left(r, r^{\prime}\right)\left[x_{j}\left(t-d_{j}\right)\right]\left(r^{\prime}\right) d r^{\prime}+\left[p_{2}(t)\right](r)\right),
\end{aligned}
$$

where, for all $i \in\{1,2\}, s \in \mathbb{R}$ and all $r \in \Omega, S_{i}(r, s):=\mathcal{S}_{i}\left(s+\mathcal{S}_{i}^{-1}\left(z_{i}^{*}(r)\right)\right)-z_{i}^{*}(r)$. With this notation, (14) is in the form of $(6)$ with state $x=\left(x_{1}, x_{2}\right):[-\bar{d},+\infty) \rightarrow \mathcal{F}^{2}$ and input $p:=\left(p_{1}, p_{2}\right) \in \mathcal{U}^{2}$. Moreover, observing that $S_{1}(r, 0)=S_{2}(r, 0)=0$ for all $r \in \Omega$, the dynamics (14) has an equilibrium at zero. 
We list below some useful properties of the functions $S_{i}, i \in\{1,2\}$. First, let $\ell_{i}>0$ denote the Lipschitz constant of $\mathcal{S}_{i}$, then it can easily be seen that, for all $r \in \Omega$ and all $a, b \in \mathbb{R},\left|S_{i}(r, a)-S_{i}(r, b)\right| \leq \ell_{i}|a-b|$. For ease of reference, we thus introduce the following terminology.

Definition 2 (Activation function) Given $\ell>0$, a function $S: \Omega \times \mathbb{R} \rightarrow \mathbb{R}$ is said to be an activation function with slope $\ell$ if, given any fixed $r \in \Omega, S(r, \cdot)$ is nondecreasing, $S(r, 0)=0$, and, for all $a, b \in \mathbb{R},|S(r, a)-S(r, b)| \leq$ $\ell|a-b|$.

It is straightforward to see that any activation function $S$ with slope $\ell>0$ satisfies, for all $r \in \Omega$,

$$
\begin{aligned}
|S(r, a)| & \leq \ell|a|, \quad \forall a \in \mathbb{R} . \\
S(r, a+b) & \leq S(r, 2 a)+S(r, 2 b), \quad \forall a, b \in \mathbb{R} .
\end{aligned}
$$

We stress that, reasoning as in the proof of (Faye and Faugeras, 2010, Theorem 3.2.1), it can be seen that the solutions of $(14 \mathrm{~b})$ exist, are unique, and are continuously differentiable over $[0 ;+\infty)$, provided that the kernels $w_{i j}$ and initial states are in $L_{2}$, delays $d_{j}$ and time constants $\tau_{j}$ are continuous, and inputs $I_{j}$ are continuous.

\subsection{ISS of the uncontrolled population}

We start by providing conditions under which the uncontrolled population (14b) is ISS with respect to inputs arising from the controlled population (14a) and possibly exogenous structures. To that aim, we analyze the following neural fields:

$$
\tau(r)[\dot{x}(t)](r)=-[x(t)](r)+S\left(r, \int_{\Omega} w\left(r, r^{\prime}\right)\left[x\left(t-d\left(r, r^{\prime}\right)\right)\right]\left(r^{\prime}\right) d r^{\prime}+[\rho(t)](r)\right) .
$$

This class of uncontrolled delayed neural fields was extensively studied in Faye and Faugeras (2010) and Veltz and Faugeras (2011), with the only difference that, in those works, the time constant $\tau$ and the activation function $S$ were assumed homogeneous, meaning independent of $r$. A bifurcation analysis was also conducted in Atay and Hutt (2004), under the additional requirement that the kernel $w$ depends only on the distance $\left|r-r^{\prime}\right|$, but allowing for higher order dynamics. From all these works, it is a known fact that the product of the Lipschitz constant $\ell$ of the activation function $S$ by the square of the $L_{2}$-norm of the kernel $w$ regulates the stability of the origin of (17) in the absence of inputs $\rho$ : if this product is smaller than 1, then the origin is asymptotically stable: see (Atay and Hutt, 2004, Theorem 2.1), (Faye and Faugeras, 2010, Theorem 4.2.3) or (Veltz and Faugeras, 2011, Proposition 3.15). Here we show that, under the same condition, the delayed neural fields (17) are actually ISS, which provides robustness with respect to the input $\rho$. The proof is provided in Section 8.4.

Proposition 1 (ISS of the uncontrolled population) Let $S$ be any activation function with slope $\ell$, let $d$ : $\Omega \times \Omega \rightarrow[0 ; \bar{d}], \tau: \Omega \rightarrow \mathbb{R}_{>0}$ and $w: \Omega \times \Omega \rightarrow \mathbb{R}$ be any bounded functions satisfying

$$
\ell \int_{\Omega} \int_{\Omega} w\left(r, r^{\prime}\right)^{2} d r^{\prime} d r<1
$$

Then (17) is ISS. Moreover, there exists $\beta: \Omega \rightarrow \mathbb{R}_{>0}$ and, for each $c>0$ small enough, there exist $c_{1}, c_{2}, c_{3}>0$ such that the functional $V: \mathcal{C} \rightarrow \mathbb{R}_{\geq 0}$ defined as

$$
V\left(x_{t}\right):=\int_{\Omega} \frac{\tau(r)}{2}[x(t)](r)^{2} d r+\int_{\Omega} \beta(r) \int_{\Omega} \int_{-d\left(r, r^{\prime}\right)}^{0} e^{c \theta}[x(t+\theta)]\left(r^{\prime}\right)^{2} d \theta d r^{\prime} d r
$$

satisfies, for all $x_{0} \in \mathcal{C}$ with continuous time derivative, all $\rho \in \mathcal{U}$, and almost all $t \in \mathbb{R}_{\geq 0}$,

$$
\dot{V}^{(17)} \leq-c_{1} V-c_{2} \int_{\Omega} \int_{\Omega}\left[x\left(t-d\left(r, r^{\prime}\right)\right)\right]\left(r^{\prime}\right)^{2} d r^{\prime} d r+c_{3}\|\rho(t)\|_{\mathcal{F}}^{2}
$$


We stress that the Krasovskii-Lyapunov functional in (19) cannot be lower-bounded by a $\mathcal{K}_{\infty}$ function of $\left\|x_{t}\right\|_{\mathcal{C}}$, thus making the results in Dashkovskiy and Mironchenko (2013) inapplicable with this particular functional. The same observation holds for the functionals considered in Proposition 2.

\subsection{ISS of the controlled population}

We now proceed to studying the controlled dynamics (14a). To this aim we consider the following class of delayed neural fields:

$$
\tau(r)[\dot{x}(t)](r)=-[x(t)](r)+S\left(r, \int_{\Omega} w\left(r, r^{\prime}\right)\left[x\left(t-d\left(r, r^{\prime}\right)\right)\right]\left(r^{\prime}\right) d r^{\prime}-k \alpha(r)[x(t)](r)+[\rho(t)](r)\right) .
$$

The next proposition states that this system can be made ISS regardless of the strength of $w$ by picking a sufficiently large gain $k$. Its proof is given in Section 8.5.

Proposition 2 (ISS of the controlled population) Let $S$ be any activation function, and let $\alpha, \tau: \Omega \rightarrow \mathbb{R}_{>0}$, $d: \Omega \rightarrow[0 ; \bar{d}], \tau: \Omega \rightarrow \mathbb{R}_{>0}$, and $w: \Omega \times \Omega \rightarrow \mathbb{R}$ be any bounded functions. Then there exists $k^{*}>0$ such that, for all $k \geq k^{*}$, the delayed neural fields (20) are ISS and there exist $c_{1}, c_{2}, c_{3}>0$, independent of $k$, such that the derivative of the functional $V: \mathcal{C} \rightarrow \mathbb{R}_{\geq 0}$ defined as

$$
V\left(x_{t}\right):=\int_{\Omega} \frac{\tau(r)}{2}[x(t)](r)^{2} d r+\int_{\Omega} \frac{\tau(r)}{2 \#_{\Omega}} \int_{\Omega} \int_{-d\left(r, r^{\prime}\right)}^{0} e^{\theta}[x(t+\theta)]\left(r^{\prime}\right)^{2} d \theta d r^{\prime} d r
$$

satisfies, for all $x_{0} \in \mathcal{C}$ with continuous time derivative, all $\rho \in \mathcal{U}$, and almost all $t \in \mathbb{R}_{\geq 0}$,

$$
\dot{V}^{(20)} \leq-c_{1} V-c_{2} \int_{\Omega} \int_{\Omega}\left[x\left(t-d\left(r, r^{\prime}\right)\right)\right]\left(r^{\prime}\right)^{2} d r^{\prime} d r+\frac{c_{3}}{k}\|\rho(t)\|_{\mathcal{F}}^{2}
$$

This result not only states that the proportional feedback (4) allows to robustly stabilize the controlled population, but also that the resulting ISS gain can be made arbitrarily small by picking $k$ large enough.

\subsection{ISS stabilization of coupled neural fields}

Proposition 1 provides conditions under which the uncontrolled population is ISS. Proposition 2 shows that the controlled population can be made ISS by the proportional feedback (4) and that an arbitrarily small ISS gain can be assigned. Based on these two results, and invoking small-gain arguments, the following result provides conditions under which the overall closed-loop neural fields (14) can be made ISS.

Proposition 3 (ISS of the whole population) For each $i, j \in\{1,2\}$, let $S_{i}$ be any activation function, and consider any bounded functions $w_{i j}: \Omega \times \Omega \rightarrow \mathbb{R}, d_{i}: \Omega \times \Omega \rightarrow[0, \bar{d}]$, and $\tau_{i}, \alpha: \Omega \rightarrow \mathbb{R}_{>0}$. Let $\ell_{2}$ denote the slope of $S_{2}$ and assume that the internal kernel $w_{22}$ of the uncontrolled population satisfies

$$
\ell_{2} \int_{\Omega} \int_{\Omega} w_{22}\left(r, r^{\prime}\right)^{2} d r^{\prime} d r<1 .
$$

Then there exists $k^{*}>0$ such that, for any $k \geq k^{*}$, the coupled neural fields (14) are ISS.

This result establishes that ISS stabilization of coupled delayed neural fields can be achieved by proportional feedback provided that the internal synaptic weights $w_{22}$ within the uncontrolled population are reasonably low. This internal synaptic coupling is allowed to be stronger if the slope of the activation function of the uncontrolled population is lower, thus indicating a trade-off between excitability and interconnection strength of the uncontrolled population. Observing that condition (22) ensures global asymptotic stability of the uncontrolled population in the absence of exogenous inputs (see Proposition 1), the above result states that proportional feedback may tackle any sustained oscillation resulting either from the interconnection between the two subpopulations or from a too strong coupling within the controlled population, but may fail at attenuating oscillations that endogenously take place within the uncontrolled population. As detailed in Detorakis et al. (2015) and recalled through simulations in Section 5, this result may prove useful to counteract pathological oscillations associated to some neurological diseases. 


\subsection{Practical stabilization by a uniform input}

As stressed in Detorakis et al. (2015), practical constraints may impede the use of a large number of independent stimulation sources. In the same way, it may not be possible to precisely measure local activity at any points of the controlled population. High spatial resolution in both measurements and actuation, in the controlled population, being a prerequisite for the application of the feedback law (4), these two practical constraints constitute severe threats to the experimental relevance of this stimulation scheme. It is therefore of interest to know whether oscillations disruption can be achieved using a limited number of stimulation points and to rely on a limited number of activity measurements. To this aim, we consider the extreme case when a single stimulation point is available and when the only available measurement is a weighted average of the activity in the controlled population. More precisely, rather than (4), we make use of the uniform feedback law (5). With this control law, the closed-loop system now reads

$$
\begin{aligned}
& \tau_{1}(r)\left[\dot{x}_{1}(t)\right](r)=-\left[x_{1}(t)\right](r)+S_{1}\left(r, \int_{\Omega} \sum_{i=1}^{2} w_{1 i}\left(r, r^{\prime}\right)\left[x_{i}\left(t-d_{i}\left(r, r^{\prime}\right)\right)\right]\left(r^{\prime}\right) d r^{\prime}-k \alpha(r) \int_{\Omega} \alpha^{\prime}\left(r^{\prime}\right)\left[x_{1}(t)\right]\left(r^{\prime}\right) d r^{\prime}\right) \\
& \tau_{2}(r)\left[\dot{x}_{2}(t)\right](r)=-\left[x_{2}(t)\right](r)+S_{2}\left(r, \int_{\Omega} \sum_{i=1}^{2} w_{2 i}\left(r, r^{\prime}\right)\left[x_{i}\left(t-d_{i}\left(r, r^{\prime}\right)\right)\right]\left(r^{\prime}\right) d r^{\prime}\right)
\end{aligned}
$$

where we may assume that $\int_{\Omega} \alpha^{\prime}(r) d r=1$ without loss of generality. The main difference with (14) stands in the fact that the control signal $-k\left[x_{1}(t)\right](r)$, which involved infinitely many stimulation and measurement points, has been replaced by the uniform control signal (5). We also removed exogenous disturbances $p_{1}$ and $p_{2}$ as these will be taken as the difference between those two stimulation signals in the proof.

In order to analyze this closed-loop system, we introduce the following measure of spatial heterogeneity: given a function $q: \Omega \rightarrow \mathbb{R}$, it is defined as

$$
\mathcal{H}(q):=\sqrt{\int_{\Omega} \int_{\Omega}\left(q(r)-q\left(r^{\prime}\right)\right)^{2} d r^{\prime} d r}
$$

Moreover, for a function $q: \Omega \times \Omega \rightarrow \mathbb{R}$, it is defined as

$$
\mathcal{H}(q):=\sqrt{\int_{\Omega} \int_{\Omega} \int_{\Omega}\left(q\left(r, r^{\prime \prime}\right)-q\left(r^{\prime}, r^{\prime \prime}\right)\right)^{2} d r^{\prime \prime} d r^{\prime} d r .}
$$

The following result shows that oscillations disruption can be achieved with the uniform control law (5), up to a steady-state error whose magnitude is somewhat proportional to the spatial heterogeneity of the controlledpopulation kernels and of the function $\alpha$.

Proposition 4 (Uniform feedback) Under the assumptions of Proposition 3, consider any $k \geq k^{*}$. Assume that the activation functions $S_{i}, i \in\{1,2\}$, are bounded and let $\underline{S}_{i}:=\inf _{x \in \mathbb{R}, r \in \Omega} S_{i}(r, x)$ and $\bar{S}_{i}:=\sup _{x \in \mathbb{R}, r \in \Omega} S_{i}(r, x)$. Assume further that the delays $d_{1}$ and $d_{2}$ and the time constant $\tau_{1}$ are uniform (meaning that $d_{1}\left(r, r^{\prime}\right)=d_{1}^{*}, d_{2}\left(r, r^{\prime}\right)=$ $d_{2}^{*}$ and $\tau_{1}(r)=\tau_{1}^{*}$ for all $\left.r, r^{\prime} \in \Omega\right)$. Then there exist $\nu_{1}, \nu_{2} \in \mathcal{K}_{\infty}$ such that, for all $x_{10} \in C\left(\left[-d_{1}^{*} ; 0\right],\left(\underline{S}_{1} ; \bar{S}_{1}\right)\right)$ and all $x_{20} \in C\left(\left[-d_{2}^{*} ; 0\right],\left(\underline{S}_{2} ; \bar{S}_{2}\right)\right)$, the solutions of $(23)$ satisfy

$$
\limsup _{t \rightarrow \infty}\|x(t)\|_{\mathcal{F}} \leq \nu_{1}\left(\mathcal{H}\left(w_{11}\right)+\mathcal{H}\left(w_{12}\right)\right)+\nu_{2}(\mathcal{H}(\alpha))
$$

This result, proved in Section 8.7, states that a single stimulation disrupts oscillations in coupled neural fields provided that the spatial heterogeneity of the kernels $w_{1 j}, j \in\{1,2\}$, of the controlled population and that of the input function $\alpha$ are sufficiently small. Practical stability is achieved, in the sense that the spatial $L_{2}$-norm of the steady-state error can be made arbitrarily small provided that those spatial heterogeneities are sufficiently small. In particular, asymptotic convergence to zero is ensured if the function $\alpha$ and the kernels $w_{1 j}$ are constant over $\Omega$. This result is established under the assumptions that the delay distributions and the time constant distribution of the controlled population are uniform and that the activation functions are bounded. We stress that this result imposes no restriction on the spatial heterogeneity of initial conditions or the kernels $w_{2 j}$. 
In practice, the real-time estimation of the controlled population activity requires some acquisition and processing time. In presence of such acquisition and processing delays, the control signal reads

$$
[u(t)](r)=-k\left[x_{1}\left(t-d_{c}(t, r)\right)\right](r)
$$

where $d_{c}: \mathbb{R}_{\geq 0} \times \Omega \rightarrow \mathbb{R}_{\geq 0}$ is a bounded function representing the feedback delay: we authorize it be time- and space-dependent for the sake of generality. With this feedback law, the closed-loop system reads

$$
\begin{aligned}
& \tau_{1}(r)\left[\dot{x}_{1}(t)\right](r)=-\left[x_{1}(t)\right](r)+S_{1}\left(\left(r, \int_{\Omega} \sum_{i=1}^{2} w_{1 i}\left(r, r^{\prime}\right)\left[x_{i}\left(t-d_{i}\left(r, r^{\prime}\right)\right)\right]\left(r^{\prime}\right) d r^{\prime}-k \alpha(r)\left[x_{1}\left(t-d_{c}(t, r)\right)\right](r)\right)\right. \\
& \tau_{2}(r)\left[\dot{x}_{2}(t)\right](r)=-\left[x_{2}(t)\right](r)+S_{2}\left(r, \int_{\Omega} \sum_{i=1}^{2} w_{2 i}\left(r, r^{\prime}\right)\left[x_{i}\left(t-d_{i}\left(r, r^{\prime}\right)\right)\right]\left(r^{\prime}\right) d r^{\prime}\right) .
\end{aligned}
$$

The only difference with (14) stands in the fact that the control signal (4) has been replaced by its delayed version (26). Similarly to the above section, we also removed exogenous disturbances $p_{1}$ and $p_{2}$ as these will be taken as the difference between those two stimulation signals in the proof (provided in Section 8.8).

Proposition 5 (Robustness to feedback delays) Under the assumptions of Proposition 3, consider any $k \geq k^{*}$. Assume further that the function $S_{1}$ is bounded and let $\underline{S}_{1}:=\inf _{x \in \mathbb{R}, r \in \Omega} S_{1}(r, x)$ and $\bar{S}_{1}:=\sup _{x \in \mathbb{R}, r \in \Omega} S_{1}(r, x)$. Then there exists a function $\nu \in \mathcal{K}_{\infty}$ such that, for all $x_{10} \in C\left([-\bar{d} ; 0],\left(\underline{S}_{1} ; \bar{S}_{1}\right)\right)$ and all $x_{20} \in \mathcal{C}$, the solutions of $(27)$ satisfy

$$
\limsup _{t \rightarrow \infty}\|x(t)\|_{\mathcal{F}} \leq \nu\left(\sup _{t \geq 0, r \in \Omega} d_{c}(t, r)\right)
$$

As already stressed in Detorakis et al. (2015); Haidar et al. (2016), the feedback delay $d_{c}$ may compromise the efficiency of the proposed feedback stimulation. The above result states that the steady-state error is arbitrarily small provided that the feedback delay is sufficiently small. In other words, if the hardware technology and the associated software authorize reasonable acquisition and processing delays, oscillations disruption will remain efficient. We stress that this result relies on the technical assumption that the activation function of the controlled population is bounded. More importantly, the asymptotic gain $\nu$ may depend on $k$, meaning that a higher feedback gain may result in a lower robustness to feedback delays.

\section{Numerical simulations}

\subsection{Parameters choice}

In order to illustrate the theoretical contributions of this paper, we simulate two spatiotemporal delayed neural fields representing the network composed of two subcortical structures, namely the STN and the GPe. As mentioned earlier, this network plays a key role in the generation of pathological oscillations related to motor symptoms in Parkinson's disease (Plenz and Kital, 1999). A spatiotemporal extension of the model Nevado-Holgado et al. (2010) was proposed in Detorakis et al. (2015), with a (mostly numerical) analysis of its behavior in the presence and absence of closed-loop stimulation. The model parameters were tuned based on data from the literature. We refer the reader to this latter reference for further biological justifications on this choice of parameters.

In this setup, the controlled population (2) is the STN whereas the uncontrolled population (3) is the GPe. The physical space $\Omega=[0,15] \mathrm{mm}$ is divided in two regions $\Omega_{1}=[0,2.5] \mathrm{mm}$ corresponding to STN, and $\Omega_{2}=[12.5,15] \mathrm{mm}$ corresponding to GPe. $I_{1}$ and $I_{2}$ are the external inputs from cortex and striatum, respectively. They are taken as white noises with variance 0.05 centered at $27 \mathrm{spk} / \mathrm{s}$ and $2 \mathrm{spk} / \mathrm{s}$, respectively. The activation functions $S_{i}, i \in\{1,2\}$, are taken as sigmoids, namely $S_{i}(x)=m_{i} b_{i} /\left(b_{i}+\left(m_{i}-b_{i}\right) e^{-4 x / m_{i}}\right)$, where $m_{1}=300 \mathrm{spk} / \mathrm{s}, b_{1}=17 \mathrm{spk} / \mathrm{s}, m_{2}=400 \mathrm{spk} / \mathrm{s}$ 
and $b_{2}=75 \mathrm{spk} / \mathrm{s}$. These functions are globally Lipschitz with Lipschitz constants $\ell_{i}=1$, which is in line with Definition 2. The delays $d_{i}$ are given by $d_{i}\left(r, r^{\prime}\right)=\frac{\left|r-r^{\prime}\right|}{c_{i}}$, for all $r, r^{\prime} \in \Omega$, where $c_{i}$ denotes the conductance velocity for neurons projecting on population $i$. Their nominal values are $c_{1}=2.5 \mathrm{~m} / \mathrm{s}$ and $c_{2}=1.4 \mathrm{~m} / \mathrm{s}$. The synaptic coupling within STN $w_{11}$ was taken as zero. The kernels $w_{12}$ and $w_{21}$, modeling synaptic weights between the two populations, were taken as $w_{12}\left(r_{1}, r_{2}\right)=-g_{12}\left(\left|r_{1}-r_{2}-\mu_{2}\right|\right)$ and $w_{21}\left(r_{2}, r_{1}\right)=g_{21}\left(\left|r_{2}-r_{1}-\mu_{1}\right|\right)$ for all $r_{1} \in \Omega_{1}, r_{2} \in \Omega_{2}$, whereas the synaptic coupling within GPe was picked as $w_{22}\left(r, r^{\prime}\right)=-\left|r-r^{\prime}\right| g_{22}\left(\left|r-r^{\prime}\right|\right)$ for all $r, r^{\prime} \in \Omega_{2}$ and zero anywhere else, where $\mu_{1}=1.25 \mathrm{~mm}$ and $\mu_{2}=13.25 \mathrm{~mm}$ indicate the centers of STN and GPe respectively, and the functions $g_{i j}$ are Gaussian functions: $g_{i j}(x):=K_{i j} \exp \left(-\frac{x^{2}}{2 \sigma_{i j}}\right)$. The nominal values of these parameters are $K_{12}=30, K_{21}=38$, $K_{22}=2.55, \sigma_{12}=\sigma_{21}=0.03$, and $\sigma_{22}=0.015$. The function $\alpha$, modeling sensitivity to light stimulation, was taken as a Gaussian function (zero outside $\Omega_{1}$ ) of amplitude 1, centered at the middle of the STN population (1.25 mm), and of variance 1.25. The source code, written in Python (Numpy,Scipy, Matplotlib), is freely distributed under the GPL License and can be found on-line at Github ${ }^{3}$.

\subsection{Stabilization by proportional feedback}

Figure 1 shows that the model reproduces sustained $\beta$-band oscillations in the absence of stimulation, as observed in the STN and GPe of parkinsonian patients (Little et al., 2016). At time $t=0.5 \mathrm{~s}$ the proportional feedback (4) is applied with gain $k=2$. As anticipated by Proposition 3, oscillations are ruled out by this proportional stimulation and the activity converges to an equilibrium configuration. This suggests that the considered distributed proportional stimulation (4) is a good candidate for the attenuation of pathological oscillations.

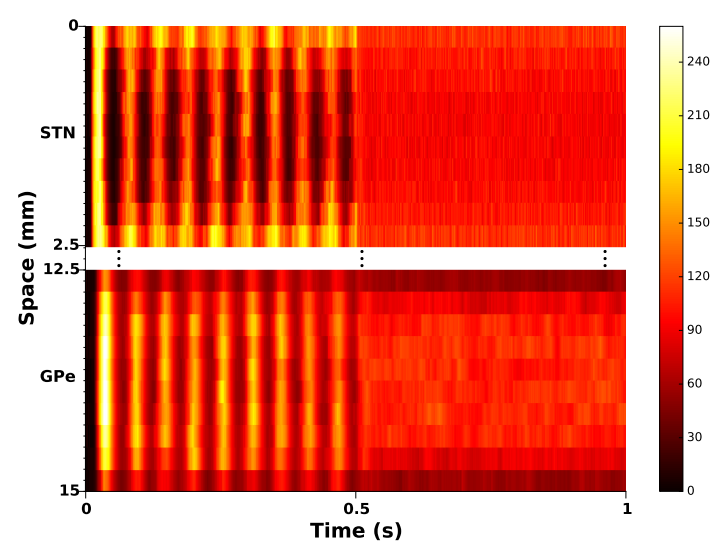

Fig. 1. Spatiotemporal evolution of STN and GPe activity. Proportional feedback (4) with $k=2$ is activated at $t=0.5 \mathrm{~s}$.

\subsection{Uniform feedback}

Figure 2 represents the magnitude of steady-state oscillations in STN (as measured though the $\mathcal{F}$-norm of $z_{1}(t)$ ) under the uniform stimulation signal (5), as a function of the feedback gain $k$. The weighting function $\alpha^{\prime}$ in $(5)$ was picked uniformly equal to 1, meaning that all neurons in STN contribute equally to the measured average activity. This figure goes beyond Proposition 4 by suggesting that the magnitude of steady-state oscillations can be arbitrarily reduced by uniform proportional feedback, provided that a sufficiently large gain $k$ is used. This observation is encouraging from a implementation viewpoint as, despite the development of holographic techniques (Reutsky-Gefen et al., 2013), uniform photostimulation remains more realistic.

\subsection{Robustness to acquisition and processing delays}

Finally, we assess the impact of acquisition and processing delays on the closed-loop performance. To this end, we consider the delayed feedback (26) with a constant and uniform delay $d_{c}$. Figure 3 shows the magnitude of STN oscillations after transients, as measured through the $\mathcal{F}$-norm of $z_{1}(t)$, for increasing valued of the feedback delay

\footnotetext{
3 https://github.com/gdetor/neuralfieldDBSmodel
} 


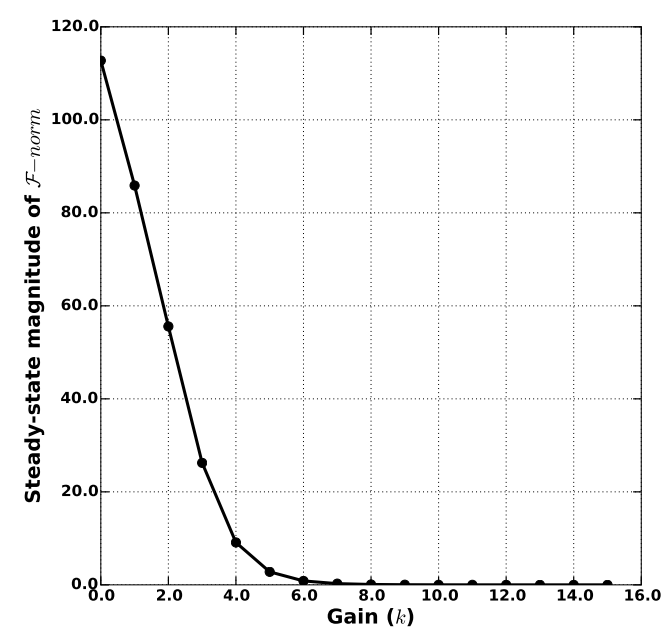

Fig. 2. Magnitude of the $\mathcal{F}$-norm of steady-state oscillations in STN as a function of the applied control gain $k$ for the uniform control law (5).

$d_{c}$. Three different values of the feedback gain $k$ were considered (namely, $k=2,6$ and 12). This figure illustrates the theoretical predictions of Proposition 5: the magnitude of steady-state oscillations is arbitrarily small provided that the feedback delay $d_{c}$ is sufficiently small. Not surprisingly, it also suggests that a better robustness to feedback delays is ensured if lower feedback gains are employed. The magnitude of steady-state oscillations in the absence of stimulation (meaning for $k=0$ ) is indicated by a red dashed line. It can be seen that, if the feedback delay $d_{c}$ is too large, then the closed-loop stimulation not only fails at attenuating pathological oscillations, but actually increases them. This observation suggests that strong attention should be paid on the delays needed to acquire and process neuronal activity in real time and pleads for the development of control strategies that allow for more robustness to feedback delays as the one proposed in Haidar et al. (2016).

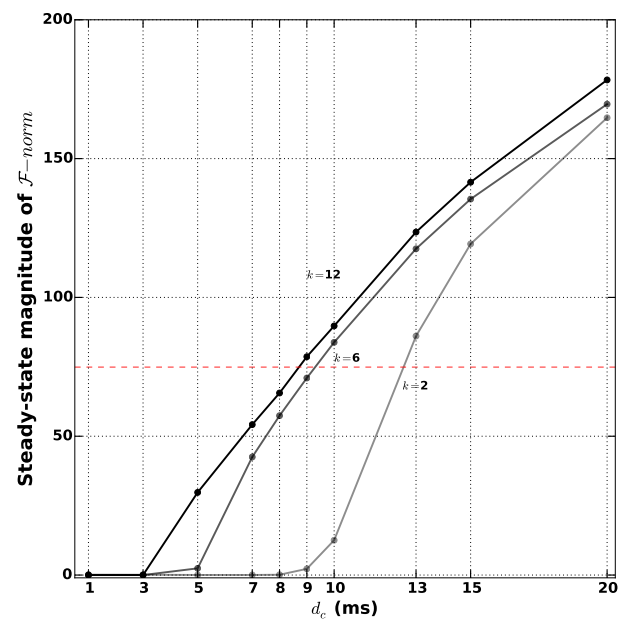

Fig. 3. Steady-state magnitude of the $\mathcal{F}$-norm of the activity in STN as a function of the feedback delay $d_{c}$ in $(26)$, for different values of the feedback gain $k$.

\section{Conclusion and perspectives}

By invoking small-gain arguments, we have shown that a simple proportional feedback, relying on partial measurement and actuation, can robustly stabilize delayed neural fields provided that the inner synaptic coupling within the uncontrolled population is reasonably low. These findings rely on an extension of the framework of input-to-state 
stability to spatiotemporal delayed dynamics. The induced robustness allowed in turn to assess the performance of the oscillations attenuation in the case when a single non-local measurement is available and when the whole controlled population is stimulated by the same input. The effect of feedback delays was also assessed. All the results were confirmed by simulation.

This work opens several lines of research. First, in the present setup, the dynamics of the actuation was not precisely taken into account. In the case of optogenetics, the impact of light impulses on neuronal activity is not immediate, but rather follows a first-order dynamics (Grossman et al., 2011). Moreover, the maximum activity generated by light stimulation is limited as neurons tend to respond less when the stimulation frequency is too high. Second, while the present paper constitutes a first stabilizability result for neural fields (even in the non-delayed case), it would be interesting to conduct a formal controllability and observability analysis of such models. Third, efficient oscillations disruption is achieved if a sufficiently large feedback gain is used. The Lyapunov methods used here impede a precise estimate of the minimum gain needed to achieve this goal. An adaptive strategy would be of practical interest. Fourth, the strategy developed here attenuates all oscillations. Finer strategies, that aim at canceling oscillations lying in a targeted frequency band would be needed. Finally, the presented small-gain results involve only two subpopulations. In our setup, this is justified by the fact that the first population is defined as the one receiving direct stimulation, whereas all other neurons are embedded in the second subpopulation. For other applications, it might however be of interest to consider more than two subpopulations, in which case large-scale small gain results, in the spirit of Dashkovskiy et al. (2010); Dashkovskiy and Mironchenko (2013), could be derived in order to reduce the conservatism of the approach.

\section{Acknowledgment}

The authors of the paper would like to thank Ihab Haidar, Elena Panteley, and William Pasillas-Lépine as the work conducted with them in the past in a non-spatial model motivated the present study. They are also thankful to Yacine Chitour, Luca Greco, Paolo Mason, Pierdomenico Pepe for useful discussions on this topic. Finally, they thank anonymous reviewers for the careful evaluation of the paper and their valuable suggestions.

\section{Proofs}

\subsection{Proof of Theorem 1}

In view of Lemma 2 , we can restrict our attention to continuously differentiable initial states $x_{0} \in \mathcal{C}$. Given any essentially bounded $p \in \mathcal{U}$ and any such initial state, let $x(\cdot)$ denote the solution of $(6)$, whose existence and uniqueness hold by assumption. Let $\bar{p}:=\operatorname{ess}_{\sup _{t>0}}\|p(t)\|_{\mathcal{F}}$ and consider the set $M:=\{\phi \in \mathcal{C}: \dot{\phi} \in \mathcal{C}, V(\phi) \leq \gamma(\bar{p})\}$. In view of Lemma 1 and since $V$ is locally Lipschitz, the function $t \mapsto V\left(x_{t}\right)$ is absolutely continuous. Since, in view of (9) and (10), $\dot{V} \leq 0$ at almost all times for which $V\left(x_{t}\right)>\gamma(\bar{p})$, the set $M$ is forward invariant, in the sense that if $x_{t_{0}} \in M$ then $x_{t} \in M$ for all $t \geq t_{0}$. In particular, observing that $\|x(t)\|_{\mathcal{F}} \leq\left\|x_{t}\right\|_{\mathcal{C}}$, it holds that

$$
\|x(t)\|_{\mathcal{F}} \leq \underline{\alpha}^{-1} \circ \gamma(\bar{p}), \quad \forall t \geq 0, \forall x_{0} \in M
$$

thus making the statement proved for all initial conditions in $M$. Now, consider any continuously differentiable $x_{0} \in \mathcal{C} \backslash M$. Let $T>0$ be any time instant such that $x_{t} \in \mathcal{C} \backslash M$ for all $t \in[0 ; T)$. Then it holds from (9) that $V\left(x_{t}\right) \geq \gamma(\bar{p})$ for all $t \in[0, T)$ and (10) therefore ensures that $\dot{V}^{(6)} \leq-\alpha\left(V\left(x_{t}\right)\right) \leq-\alpha \circ \bar{\alpha}^{-1}\left(V\left(x_{t}\right)\right)$ for almost all $t \in[0, T)$. Invoking (Mironchenko and Ito, 2016, Lemma 3.2) it follows that there exists $\tilde{\beta} \in \mathcal{K} \mathcal{L}$ (depending only on $\alpha$ and $\bar{\alpha})$ such that $V\left(x_{t}\right) \leq \tilde{\beta}\left(V\left(x_{0}\right), t\right)$ for all $t \in[0, T)$. We conclude from $(9)$ that

$$
\|x(t)\|_{\mathcal{F}} \leq \beta\left(\left\|x_{0}\right\|_{\mathcal{C}}, t\right), \quad \forall t \in[0 ; T)
$$

where $\beta(s, t):=\underline{\alpha}^{-1} \circ \tilde{\beta}(\bar{\alpha}(s), t)$ for all $s, t \geq 0$. If the supremum of all such $T$ 's is infinity, then the above estimate holds for all $t \in[0 ;+\infty)$ and the conclusion follows. Otherwise, it necessarily holds that $x_{T} \in M$ for some finite $T>0$ and we conclude from (28) and (29) that $\|x(t)\|_{\mathcal{F}} \leq \beta\left(\left\|x_{0}\right\|_{\mathcal{C}}, t\right)+\underline{\alpha}^{-1} \circ \gamma(\bar{p})$ for all $t \geq 0$, which concludes the proof due to causality. 


\subsection{Proof of Theorem 2 (sketch)}

One strength of the proofs of the main results in Jiang et al. (1996) is that they mostly rely on the functions $\underline{\alpha}_{i}, \bar{\alpha}_{i}, \gamma_{i}$ and $\chi_{i}$ : the fact that the arguments of $V_{i}$ are vectors or functions plays no significant role. Consequently, those results can easily be extended to Lyapunov functionals. More precisely, following the steps of the proof of (Jiang et al., 1996, Theorem 3.1), one can construct a locally Lipschitz functional $V \in C\left(\mathcal{C}^{n_{1}+n_{2}}, \mathbb{R}_{\geq 0}\right)$ and $\underline{\alpha}, \bar{\alpha}, \alpha, \gamma \in \mathcal{K}_{\infty}$ such that

$$
\begin{array}{r}
\underline{\alpha}\left(\|x(t)\|_{\mathcal{F}}\right) \leq V\left(x_{t}\right) \leq \bar{\alpha}\left(\left\|x_{t}\right\|_{\mathcal{C}}\right) \\
V\left(x_{t}\right) \geq \gamma\left(\|p(t)\|_{\mathcal{F}}\right) \Rightarrow \dot{V}^{(11)} \leq-\alpha(V)
\end{array}
$$

where $x:=\left(x_{1}, x_{2}\right)$ and $p:=\left(p_{1}, p_{2}\right)$. The conclusion then follows from Theorem 1 .

\subsection{Proof of Corollary 1}

Let $\sigma:=\frac{k_{21}}{2}+\frac{1}{2 k_{12}}$. Then, under the small-gain condition $k_{12} k_{21} \leq 1$, it holds that $k_{21} \leq \sigma \leq \frac{1}{k_{12}}$. Consider the functional defined as $V\left(x_{t}\right):=\sigma V_{1}\left(x_{1 t}\right)+V_{2}\left(x_{2 t}\right)$, where $x_{t}:=\left(x_{1 t}, x_{2 t}\right)$. Then $V$ satisfies $(12)$ with $\underline{\alpha}(s):=$ $\min \left\{\sigma \underline{\alpha}_{1}(s / \sqrt{2}) ; \underline{\alpha}_{2}(s / \sqrt{2})\right\}$ for all $s \in \mathbb{R}_{\geq 0}$ (as can be seen by considering separately the cases $\left\|x_{1}(t)\right\|_{\mathcal{F}} \geq\left\|x_{2}(t)\right\|_{\mathcal{F}}$ and $\left.\left\|x_{1}(t)\right\|_{\mathcal{F}} \leq\left\|x_{2}(t)\right\|_{\mathcal{F}}\right)$ and $\bar{\alpha}:=\sigma \bar{\alpha}_{1}+\bar{\alpha}_{2}$. Moreover its derivative reads, for almost all $t \geq 0$,

$$
\begin{aligned}
\dot{V}^{(11)} & =\sigma \dot{V}_{1}^{(11 \mathrm{a})}+\dot{V}_{2}^{(11 \mathrm{~b})} \\
& \leq-\sigma \alpha_{1}\left(V_{1}\right)-\alpha_{2}\left(V_{2}\right)-\left(\sigma-k_{21}\right) W_{1}-\left(1-\sigma k_{12}\right) W_{2}+\sigma \gamma_{1}\left(\left\|p_{1}(t)\right\|_{\mathcal{F}}\right)+\gamma_{2}\left(\left\|p_{2}(t)\right\|_{\mathcal{F}}\right) \\
& \leq-\sigma \alpha_{1}\left(V_{1}\right)-\alpha_{2}\left(V_{2}\right)+\gamma\left(\|p(t)\|_{\mathcal{F}}\right),
\end{aligned}
$$

where $\gamma:=\sigma \gamma_{1}+\gamma_{2}$ is clearly a $\mathcal{K}_{\infty}$ function. Let $\alpha(s):=\min \left\{\sigma \alpha_{1}(s / 2 \sigma) ; \alpha_{2}(s / 2)\right\}$ for all $s \in \mathbb{R}_{>0}$, then $\alpha$ is also a $\mathcal{K}_{\infty}$ function and it holds that $\alpha(V)=\alpha\left(\sigma V_{1}+V_{2}\right) \leq \alpha\left(2 \sigma V_{1}\right)+\alpha\left(2 V_{2}\right) \leq \sigma \alpha_{1}\left(V_{1}\right)+\alpha_{2}\left(V_{2}\right)$. It follows that $\dot{V}^{(11)} \leq-\alpha(V)+\gamma\left(\|p(t)\|_{\mathcal{F}}\right)$. In particular, the following implication holds for almost all $t \geq 0$ :

$$
V\left(x_{t}\right) \geq \alpha^{-1} \circ 2 \gamma\left(\|p(t)\|_{\mathcal{F}}\right) \quad \Rightarrow \quad \dot{V}^{(11)} \leq-\frac{1}{2} \alpha\left(V\left(x_{t}\right)\right)
$$

which concludes the proof in view of Theorem 1.

\subsection{Proof of Proposition 1}

In order to avoid cumbersome notation, we will omit to write the arguments of some functions in the proof. In particular, it should be kept in mind that the delay $d$ and the kernel $w$ depends on both $r$ and $r^{\prime}$. Also, unless explicitly specified, we will denote $[x(t)](r)$ (resp. $[\rho(t)](r))$ as simply $x$ (resp. $\rho$ ). In the same way, $S(r, x)$ will simply be denoted as $S(x)$. First notice that the right-hand side of (17) is globally Lipschitz, ensuring forward completeness and uniqueness of solutions (see also Faye and Faugeras (2010)). Moreover, the functional $V$ defined in (19) is locally Lipschitz. Considering any continuously differentiable $x_{0} \in \mathcal{C}$, it follows from Lemma 1 that $t \mapsto V\left(x_{t}\right)$ is absolutely continuous. We decompose $V$ as $V\left(x_{t}\right)=V_{a}(x(t))+V_{b}\left(x_{t}\right)$ where

$$
\begin{aligned}
V_{a}(x(t)) & :=\int_{\Omega} \frac{\tau(r)}{2}[x(t)](r)^{2} d r \\
V_{b}\left(x_{t}\right) & :=\int_{\Omega} \beta(r) \int_{\Omega} \int_{-d\left(r, r^{\prime}\right)}^{0} e^{c \theta}[x(t+\theta)]\left(r^{\prime}\right)^{2} d \theta d r^{\prime} d r .
\end{aligned}
$$

With the variable change $\theta \leftarrow t+\theta$, it can be seen that the time derivative of $V_{b}$ reads, for almost all $t \geq 0$,

$$
\begin{aligned}
\dot{V}_{b}^{(17)} & =\int_{\Omega} \beta(r) \int_{\Omega}\left([x(t)]\left(r^{\prime}\right)^{2}-e^{-c d}[x(t-d)]\left(r^{\prime}\right)^{2}\right) d r^{\prime} d r-c V_{b} \\
& \leq \int_{\Omega} \beta(r) d r \int_{\Omega}[x(t)](r)^{2} d r-e^{-c \bar{d}} \int_{\Omega} \int_{\Omega}\left[x\left(t-d\left(r, r^{\prime}\right)\right)\right]\left(r^{\prime}\right)^{2} d r^{\prime} d r-c V_{b} .
\end{aligned}
$$


Furthermore, the derivative of $V_{a}$ can be computed as follows. First, exploiting the fact that $|S(r, a)| \leq \ell|a|$ for all $a \in \mathbb{R}$ and all $r \in \Omega$, as recalled in (15), it holds that

$$
\begin{aligned}
\dot{V}_{a}^{(17)} & =-\int_{\Omega}[x(t)](r)^{2} d r+\int_{\Omega}[x(t)](r) S\left(r, \int_{\Omega} w\left(r, r^{\prime}\right)\left[x\left(t-d\left(r, r^{\prime}\right)\right)\right]\left(r^{\prime}\right) d r^{\prime}+[\rho(t)](r)\right) d r \\
& \leq-\int_{\Omega} x^{2} d r+\ell \int_{\Omega}|x|\left|\int_{\Omega} w x(t-d) d r^{\prime}+\rho\right| d r \\
& \leq-\int_{\Omega} x^{2} d r+\ell \int_{\Omega} \int_{\Omega}|x||w||x(t-d)| d r^{\prime} d r+\ell \int_{\Omega}|x||\rho| d r .
\end{aligned}
$$

Using the fact $a b \leq \frac{1}{2}\left(\lambda a^{2}+\frac{b^{2}}{\lambda}\right)$ for all $a, b \geq 0$ and all $\lambda>0$, it follows that for any functions $\lambda_{1}, \lambda_{2}: \Omega \rightarrow \mathbb{R}_{>0}$,

$$
\begin{aligned}
\dot{V}_{a}^{(17)} & \leq-\int_{\Omega} x^{2} d r+\int_{\Omega} \frac{\ell}{2 \lambda_{1}(r)} \int_{\Omega} x^{2} w^{2} d r^{\prime} d r+\int_{\Omega} \frac{\ell \lambda_{1}(r)}{2} \int_{\Omega} x(t-d)^{2} d r^{\prime} d r+\int_{\Omega} \frac{\ell}{2 \lambda_{2}(r)} x^{2} d r+\int_{\Omega} \frac{\ell \lambda_{2}(r)}{2} \rho^{2} d r \\
& \leq-\int_{\Omega}\left(1-\frac{\ell}{2 \lambda_{1}} \int_{\Omega} w^{2} d r^{\prime}-\frac{\ell}{2 \lambda_{2}}\right) x^{2} d r+\int_{\Omega} \frac{\ell \lambda_{1}}{2} \int_{\Omega} x(t-d)^{2} d r^{\prime} d r+\int_{\Omega} \frac{\ell \lambda_{2}}{2} \rho^{2} d r \\
& \leq-\int_{\Omega}\left(1-\frac{\bar{w}}{2 \lambda_{1}}-\frac{\ell}{2 \lambda_{2}}\right) x^{2} d r+\int_{\Omega} \frac{\ell \lambda_{1}}{2} \int_{\Omega} x(t-d)^{2} d r^{\prime} d r+\int_{\Omega} \frac{\ell \lambda_{2}}{2} \rho^{2} d r
\end{aligned}
$$

where $\bar{w}: \Omega \rightarrow \mathbb{R}_{>0}$ denotes any function satisfying

$$
\bar{w}(r) \geq \ell \int_{\Omega} w\left(r, r^{\prime}\right)^{2} d r^{\prime}, \quad \forall r \in \Omega
$$

Under condition (18), $\bar{w}$ can be picked in such a way that

$$
\int_{\Omega} \bar{w}(r) d r<1
$$

This can be seen by picking for instance $\bar{w}(r)=\frac{\varepsilon}{2 \# \Omega}+\ell \int_{\Omega} w\left(r, r^{\prime}\right)^{2} d r^{\prime}$, where $\varepsilon:=1-\ell \int_{\Omega} \int_{\Omega} w\left(r, r^{\prime}\right)^{2} d r^{\prime} d r$.

We claim that the proposition holds with $\beta(r)=\bar{w}(r) / 2$, where $\bar{w}$ is any positive function satisfying (34) and (35). With this choice, recalling that $V=V_{a}+V_{b}$, the combination of (32) and (33) yields

$$
\dot{V}^{(17)} \leq-\int_{\Omega}\left(1-\frac{\bar{w}}{2 \lambda_{1}}-\frac{\ell}{2 \lambda_{2}}-\int_{\Omega} \frac{\bar{w}}{2} d r^{\prime}\right) x^{2} d r-\int_{\Omega}\left(e^{-c \bar{d}}-\frac{\ell \lambda_{1}}{2}\right) \int_{\Omega} x(t-d)^{2} d r^{\prime} d r-c V_{b}+\int_{\Omega} \frac{\ell \lambda_{2}}{2} \rho^{2} d r
$$

Thus, the result is proved if we can find positive functions $\lambda_{1}, \lambda_{2}$ and a constant $c>0$ such that

$$
\frac{\bar{w}}{2 \lambda_{1}(r)}+\frac{\ell}{2 \lambda_{2}(r)}+\int_{\Omega} \frac{\bar{w}\left(r^{\prime}\right)}{2} d r^{\prime}<1, \quad e^{-c \bar{d}}>\frac{\ell \lambda_{1}(r)}{2}
$$

It can be checked that these conditions are fulfilled with

$$
\begin{aligned}
\lambda_{1}(r) & =\frac{\bar{w}(r)\left(3-\int_{\Omega} w\left(r^{\prime}\right) d r^{\prime}\right)}{2\left(2-\int_{\Omega} \bar{w}\left(r^{\prime}\right) d r^{\prime}\right)} \\
\lambda_{2}(r) & =\lambda_{2}^{*}>\frac{\ell}{2-\bar{w}(r) \lambda_{1}(r)-\int_{\Omega} \bar{w}\left(r^{\prime}\right) d r^{\prime}} \\
c & \leq \frac{1}{\bar{d}} \ln \left(\frac{1}{\ell \lambda_{1}(r)}\right) .
\end{aligned}
$$


It follows from (36) that there exists $c_{1}^{\prime}>0$ such that

$$
\begin{aligned}
\dot{V}^{(17)} & \leq-c_{1}^{\prime} \int_{\Omega} x^{2} d r-c V_{b}-\frac{e^{-c \bar{d}}}{2} \int_{\Omega} \int_{\Omega} x(t-d)^{2} d r^{\prime} d r+\frac{\ell \lambda_{2}^{*}}{2}\|\rho\|_{\mathcal{F}}^{2} \\
& \leq-\frac{2 c_{1}^{\prime}}{\bar{\tau}} V_{a}-c V_{b}-\frac{e^{-c \bar{d}}}{2} \int_{\Omega} \int_{\Omega} x(t-d)^{2} d r^{\prime} d r+\frac{\ell \lambda_{2}^{*}}{2}\|\rho\|_{\mathcal{F}}^{2}
\end{aligned}
$$

where $\bar{\tau}:=\max _{r \in \Omega} \tau(r)$. The conclusion follows by letting $c_{1}=\min \left\{2 c_{1}^{\prime} / \bar{\tau} ; c\right\}, c_{2}=e^{-c \bar{d}} / 2$, and $c_{3}=\ell \lambda_{2}^{*} / 2$.

\subsection{Proof of Proposition 2}

For the sake of readability, we rely on the same notation simplifications as the ones used in the proof of Proposition 1 everywhere the context is explicit enough. We decompose $V$ in $(21)$ as $V\left(x_{t}\right)=V_{a}(x(t))+V_{b}\left(x_{t}\right)$ where

$$
\begin{aligned}
V_{a}(x(t)) & :=\int_{\Omega} \frac{\tau(r)}{2}[x(t)](r)^{2} d r \\
V_{b}\left(x_{t}\right) & :=\frac{1}{2 \#_{\Omega}} \int_{\Omega} \int_{\Omega} \int_{-d\left(r, r^{\prime}\right)}^{0} e^{\theta}[x(t+\theta)]\left(r^{\prime}\right)^{2} d \theta d r^{\prime} d r .
\end{aligned}
$$

Reasoning as in the proof of Proposition 1, the map $t \mapsto V\left(x_{t}\right)$ is absolutely continuous for any continuously differentiable initial condition. The derivative of $V_{a}$ along the solutions of (20) reads, for almost all $t \geq 0$,

$$
\begin{aligned}
\dot{V}_{a}^{(20)} & =-\int_{\Omega}[x(t)](r)^{2} d r+\int_{\Omega}[x(t)](r) S\left(r, \int_{\Omega} w\left(r, r^{\prime}\right)\left[x\left(t-d\left(r, r^{\prime}\right)\right)\right]\left(r^{\prime}\right) d r^{\prime}-k \alpha(r)[x(t)](r)+[\rho(t)](r)\right) d r \\
& =-\int_{\Omega} x^{2} d r+\int_{\Omega} x S\left(\int_{\Omega} w[x(t-d)]\left(r^{\prime}\right) d r^{\prime}-k \alpha x+\rho\right) d r
\end{aligned}
$$

Let $\underline{\alpha}:=\min _{r \in \Omega} \alpha(r)>0$. We consider two cases. First, consider the case when $k \underline{\alpha}|x| \geq\left|\int_{\Omega} w[x(t-d)]\left(r^{\prime}\right) d r^{\prime}+\rho\right|$. Recalling that $S(r, \cdot)$ is nondecreasing and has the same sign as its argument for all $r \in \Omega$, it holds that $S(a+b)$ has the same sign as $a$ whenever $|a| \geq|b|$. Consequently,

$$
x S\left(\int_{\Omega} w[x(t-d)]\left(r^{\prime}\right) d r^{\prime}-k \alpha x+p\right) \leq 0 .
$$

On the other hand, when $k \underline{\alpha}|x|<\left|\int_{\Omega} w[x(t-d)]\left(r^{\prime}\right) d r^{\prime}+\rho\right|$, the fact that $|S(x)| \leq \ell|x|$, where $\ell$ is the slope of $S$ (see (15)), ensures that

$$
\begin{aligned}
x S\left(\int_{\Omega} w[x(t-d)]\left(r^{\prime}\right) d r^{\prime}-k x+\rho\right) & \leq|x|\left|S\left(\int_{\Omega} w[x(t-d)]\left(r^{\prime}\right) d r^{\prime}-k \underline{\alpha} x+\rho\right)\right| \\
& \leq \ell|x|\left|\int_{\Omega} w[x(t-d)]\left(r^{\prime}\right) d r^{\prime}-k \underline{\alpha} x+\rho\right| \\
& \leq \ell|x|\left(\left|\int_{\Omega} w[x(t-d)]\left(r^{\prime}\right) d r^{\prime}\right|+k \underline{\alpha}|x|+|\rho|\right) \\
& \leq \frac{\ell}{\alpha k}\left|\int_{\Omega} w[x(t-d)]\left(r^{\prime}\right) d r^{\prime}+\rho\right|\left(2\left|\int_{\Omega} w[x(t-d)]\left(r^{\prime}\right) d r^{\prime}\right|+|\rho|\right) \\
& \leq \frac{2 \ell}{\alpha k}\left(\left|\int_{\Omega} w[x(t-d)]\left(r^{\prime}\right) d r^{\prime}\right|+|\rho|\right) \\
& \leq \frac{4 \ell}{\alpha k}\left(\left|\int_{\Omega} w[x(t-d)]\left(r^{\prime}\right) d r^{\prime}\right|^{2}+\rho^{2}\right) \\
& \leq \frac{\underline{\alpha}}{\underline{\alpha} k}\left(\int_{\Omega} w^{2} d r^{\prime} \int_{\Omega}[x(t-d)]\left(r^{\prime}\right)^{2} d r^{\prime}+\rho^{2}\right)
\end{aligned}
$$


where we used Cauchy-Schwarz inequality for the latter step. In view of (40), we conclude that this bound (41) holds in any case. Plugging it into (39), we get that

$$
\dot{V}_{a}^{(20)} \leq-\int_{\Omega} x^{2} d r+\frac{4 \ell}{\underline{\alpha} k} \int_{\Omega}\left(\int_{\Omega} w^{2} d r^{\prime} \int_{\Omega}[x(t-d)]\left(r^{\prime}\right)^{2} d r^{\prime}+\rho^{2}\right) d r .
$$

Let us now move to the computation of the functional $V_{b}$ introduced in (38). Proceeding as in the proof of Proposition 1 (see (38) and subsequent equations), we get that

$$
\begin{aligned}
\dot{V}_{b}^{(20)} & =\frac{1}{2 \# \Omega} \int_{\Omega} \int_{\Omega}[x(t)]\left(r^{\prime}\right)^{2} d r^{\prime} d r-\frac{1}{2 \# \Omega} \int_{\Omega} \int_{\Omega} e^{-d\left(r, r^{\prime}\right)}\left[x\left(t-d\left(r, r^{\prime}\right)\right)\right]\left(r^{\prime}\right)^{2} d r^{\prime} d r-V_{b}\left(x_{t}\right) \\
& \leq \frac{1}{2} \int_{\Omega} x^{2} d r-\frac{e^{-\bar{d}}}{2 \# \Omega} \int_{\Omega} \int_{\Omega}[x(t-d)]\left(r^{\prime}\right)^{2} d r^{\prime} d r-V_{b} .
\end{aligned}
$$

Combining this with (42), we get that the derivative of the functional $V=V_{a}+V_{b}$ satisfies

$$
\begin{aligned}
\dot{V}^{(20)} & \leq-\frac{1}{2} \int_{\Omega} x^{2} d r-V_{b}-\int_{\Omega}\left(\frac{e^{-\bar{d}}}{2 \#_{\Omega}}-\frac{4 \ell}{\underline{\alpha} k} \int_{\Omega} w^{2} d r^{\prime}\right)[x(t-d)]\left(r^{\prime}\right)^{2} d r^{\prime} d r+\frac{4 \ell}{\underline{\alpha} k} \int_{\Omega} \rho^{2} d r \\
& \leq-\frac{1}{\bar{\tau}} V_{a}-V_{b}+\frac{4 \ell}{\underline{\alpha} k}\|\rho(t)\|_{\mathcal{F}}^{2}-\int_{\Omega}\left(\frac{e^{-\bar{d}}}{2 \# \Omega}-\frac{4 \ell}{\underline{\alpha} k} \int_{\Omega} w^{2} d r^{\prime}\right)[x(t-d)]\left(r^{\prime}\right)^{2} d r^{\prime} d r
\end{aligned}
$$

where $\bar{\tau}:=\max _{r \in \Omega} \tau(r)$. Thus, by picking $k \geq k^{*}:=\frac{16 \ell \# \Omega e^{\bar{d}}}{\underline{\alpha}} \max _{r \in \Omega} \int_{\Omega} w\left(r, r^{\prime}\right) d r^{\prime}$ and by letting $c_{1}:=\min \{1 / \bar{\tau} ; 1\}$, $c_{2}:=\frac{e^{\bar{d}}}{4 \# \Omega}$ and $c_{3}=4 \ell / \underline{\alpha}$, we obtain

$$
\dot{V}^{(20)} \leq-c_{1} V-c_{2} \int_{\Omega} \int_{\Omega}[x(t-d)]\left(r^{\prime}\right)^{2} d r^{\prime} d r+\frac{c_{3}}{k}\|\rho(t)\|_{\mathcal{F}}^{2}
$$

\subsection{Proof of Proposition 3}

First note that the subsystems (14a) and (14b) can be respectively written in the form of the systems addressed by Propositions 2 and 1 , namely (20) and (17), by considering the inputs $\rho_{1}, \rho_{2} \in \mathcal{U}$ defined, for all $t \in \mathbb{R}_{\geq 0}$, as

$$
\begin{aligned}
\rho_{1}(t) & :=\int_{\Omega} w_{12}\left(\cdot, r^{\prime}\right)\left[x_{2}\left(t-d_{2}\left(\cdot, r^{\prime}\right)\right)\right]\left(r^{\prime}\right) d r^{\prime}+p_{1}(t) \\
\rho_{2}(t) & :=\int_{\Omega} w_{21}\left(\cdot, r^{\prime}\right)\left[x_{1}\left(t-d_{1}\left(\cdot, r^{\prime}\right)\right)\right]\left(r^{\prime}\right) d r^{\prime}+p_{2}(t) .
\end{aligned}
$$

Under the assumptions of Proposition 3, those of Propositions 2 and 1 are satisfied for each relevant subsystem, and we get that there exist globally Lipschitz functionals $V_{1}, V_{2}: \mathcal{C} \rightarrow \mathbb{R}_{\geq 0}$ (respectively defined as in (21) and (19)) and $c_{i j}>0, i \in\{1,2\}, j \in\{1,2,3\}$, such that, for all $k>0$ large enough, and almost all $t \geq 0$,

$$
\begin{aligned}
\dot{V}_{1}^{(14 \mathrm{a})} & \leq-c_{11} V_{1}-c_{12} \int_{\Omega} \int_{\Omega}\left[x_{1}\left(t-d_{1}\right)\right]\left(r^{\prime}\right)^{2} d r^{\prime} d r+\frac{c_{13}}{k}\left\|\rho_{1}(t)\right\|_{\mathcal{F}}^{2} \\
\dot{V}_{2}^{(14 \mathrm{~b})} & \leq-c_{21} V_{2}-c_{22} \int_{\Omega} \int_{\Omega}\left[x_{2}\left(t-d_{2}\right)\right]\left(r^{\prime}\right)^{2} d r^{\prime} d r+c_{23}\left\|\rho_{2}(t)\right\|_{\mathcal{F}}^{2} .
\end{aligned}
$$


In view of (43) and using Cauchy-Schwarz inequality,

$$
\begin{aligned}
\left\|\rho_{1}(t)\right\|_{\mathcal{F}}^{2} & =\int_{\Omega}\left(\int_{\Omega} w_{12}\left(r, r^{\prime}\right)\left[x_{2}\left(t-d_{2}\left(r, r^{\prime}\right)\right)\right]\left(r^{\prime}\right) d r^{\prime}+\left[p_{1}(t)\right](r)\right)^{2} d r \\
& \leq 2 \int_{\Omega}\left(\int_{\Omega} w_{12}\left(r, r^{\prime}\right)\left[x_{2}\left(t-d_{2}\left(r, r^{\prime}\right)\right)\right]\left(r^{\prime}\right) d r^{\prime}\right)^{2} d r+2 \int_{\Omega}\left[p_{1}(t)\right](r)^{2} d r \\
& \leq 2 \int_{\Omega}\left(\int_{\Omega} w_{12}\left(r, r^{\prime}\right)^{2} d r^{\prime} \int_{\Omega}\left[x_{2}\left(t-d_{2}\left(r, r^{\prime}\right)\right)\right]\left(r^{\prime}\right)^{2} d r^{\prime}\right) d r+2\left\|p_{1}(t)\right\|_{\mathcal{F}}^{2} \\
& \leq 2 \overline{\bar{w}}_{12} \int_{\Omega} \int_{\Omega}\left[x_{2}\left(t-d_{2}\left(r, r^{\prime}\right)\right)\right]\left(r^{\prime}\right)^{2} d r^{\prime} d r+2\left\|p_{1}(t)\right\|_{\mathcal{F}}^{2},
\end{aligned}
$$

where $\overline{\bar{w}}_{12}:=\max _{r \in \Omega} \int_{\Omega} w_{12}\left(r, r^{\prime}\right)^{2} d r^{\prime}$. In the same way, defining $\overline{\bar{w}}_{21}:=\max _{r \in \Omega} \int_{\Omega} w_{21}\left(r, r^{\prime}\right)^{2} d r^{\prime}$, we get that

$$
\left\|\rho_{2}(t)\right\|_{\mathcal{F}}^{2} \leq 2 \overline{\bar{w}}_{21} \int_{\Omega} \int_{\Omega}\left[x_{1}\left(t-d_{1}\left(r, r^{\prime}\right)\right)\right]\left(r^{\prime}\right)^{2} d r^{\prime} d r+2\left\|p_{2}(t)\right\|_{\mathcal{F}}^{2}
$$

Plugging these bounds into (45)-(46) yields

$$
\begin{aligned}
& \dot{V}_{1}^{(14 \mathrm{a})} \leq-c_{11} V_{1}-c_{12} \int_{\Omega} \int_{\Omega}\left[x_{1}\left(t-d_{1}\right)\right]\left(r^{\prime}\right)^{2} d r^{\prime} d r+\frac{2 c_{13} \overline{\bar{w}}_{12}}{k} \int_{\Omega} \int_{\Omega}\left[x_{2}\left(t-d_{2}\right)\right]\left(r^{\prime}\right)^{2} d r^{\prime} d r+\frac{2 c_{13}}{k}\left\|p_{1}(t)\right\|_{\mathcal{F}}^{2} \\
& \dot{V}_{2}^{(14 \mathrm{~b})} \leq-c_{21} V_{2}-c_{22} \int_{\Omega} \int_{\Omega}\left[x_{2}\left(t-d_{2}\right)\right]\left(r^{\prime}\right)^{2} d r^{\prime} d r+2 c_{23} \overline{\bar{w}}_{21} \int_{\Omega} \int_{\Omega}\left[x_{1}\left(t-d_{1}\right)\right]\left(r^{\prime}\right)^{2} d r^{\prime} d r+2 c_{23}\left\|p_{2}(t)\right\|_{\mathcal{F}}^{2} .
\end{aligned}
$$

Letting $W_{1}\left(x_{1 t}\right):=c_{12} \int_{\Omega} \int_{\Omega}\left[x_{1}\left(t-d_{1}\left(r, r^{\prime}\right)\right)\right]\left(r^{\prime}\right)^{2} d r^{\prime} d r$ and $W_{2}\left(x_{2 t}\right):=c_{22} \int_{\Omega} \int_{\Omega}\left[x_{2}\left(t-d_{2}\left(r, r^{\prime}\right)\right)\right]\left(r^{\prime}\right)^{2} d r^{\prime} d r$, we finally get that

$$
\begin{aligned}
& \dot{V}_{1}^{(14 \mathrm{a})} \leq-c_{11} V_{1}-W_{1}+\frac{2 c_{13} \overline{\bar{w}}_{12}}{k c_{22}} W_{2}+\frac{2 c_{13}}{k}\left\|p_{1}(t)\right\|_{\mathcal{F}}^{2} \\
& \dot{V}_{2}^{(14 \mathrm{~b})} \leq-c_{21} V_{2}-W_{2}+\frac{2 c_{23} \overline{\bar{w}}_{21}}{c_{12}} W_{1}+2 c_{23}\left\|p_{2}(t)\right\|_{\mathcal{F}}^{2} .
\end{aligned}
$$

Invoking Corollary 1 , the system is thus ISS provided that $4 c_{13} c_{23} \overline{\bar{w}}_{12} \overline{\bar{w}}_{21} / k c_{12} c_{22} \leq 1$, which can always be satisfied by picking $k$ sufficiently large.

\subsection{Proof of Proposition 4}

The proof is made of two steps. The first one (Claim 1) consists in showing that the spatial heterogeneity of solutions is eventually small provided that the spatial heterogeneity of the kernels $w_{1 i}, i \in\{1,2\}$, and of the function $\alpha$ is sufficiently small. The second step (Claim 2) consists in showing that, if the spatial heterogeneity of solutions is small, then the difference between the original control signal (4) and the one considered here (5) is small too. For each $i \in\{1,2\}$, let $\ell_{i}>0$ denote the slope of $S_{i}$ and $\overline{\bar{S}}_{i}:=\max \left\{\left|\underline{S}_{i}\right| ;\left|\bar{S}_{i}\right|\right\}$. Let also $\overline{\bar{S}}:=\max \left\{\overline{\bar{S}}_{1} ; \overline{\bar{S}}_{2}\right\}$.

Claim 1 Under the assumptions of Proposition 4, the spatial heterogeneity of solutions of (23) satisfies, for all $t_{0} \geq 0$ and all $t \geq t_{0}$

$$
\mathcal{H}\left(x_{1}(t)\right)^{2} \leq \mathcal{H}\left(x_{1}\left(t_{0}\right)\right)^{2} e^{-\left(t-t_{0}\right) / \tau_{1}^{*}}+\frac{2 \ell_{1}^{2} \overline{\bar{S}}^{2} \#_{\Omega}}{\tau_{1}^{*}}\left(2 \sum_{i=1}^{2} \mathcal{H}\left(w_{1 i}\right)^{2}+k\left\|\alpha^{\prime}\right\|_{\mathcal{F}}^{2} \mathcal{H}(\alpha)^{2}\right)
$$


Proof In view of (24), consider the functional $W: \mathcal{F} \rightarrow \mathbb{R}_{\geq 0}$ defined as $W\left(x_{1}(t)\right):=\frac{\tau_{1}^{*}}{2} \mathcal{H}\left(x_{1}(t)\right)^{2}$. Its derivative along the solutions of (23a) reads

$$
\begin{aligned}
& \dot{W}^{(23 \mathrm{a})}=-\frac{2}{\tau_{1}^{*}} W+\int_{\Omega} \int_{\Omega}\left(\left[x_{1}(t)\right](r)-\left[x_{1}(t)\right]\left(r^{\prime}\right)\right) \\
& \times\left[S_{1}\left(r, \int_{\Omega} \sum_{i=1}^{2} w_{1 i}\left(r, r^{\prime \prime}\right)\left[x_{i}\left(t-d_{i}^{*}\right)\right]\left(r^{\prime \prime}\right) d r^{\prime \prime}-k \alpha(r) \int_{\Omega} \alpha^{\prime}\left(r^{\prime \prime}\right)\left[x_{1}(t)\right]\left(r^{\prime \prime}\right) d r^{\prime \prime}\right)\right. \\
& \left.-S_{1}\left(r^{\prime}, \int_{\Omega} \sum_{i=1}^{2} w_{1 i}\left(r^{\prime}, r^{\prime \prime}\right)\left[x_{i}\left(t-d_{i}^{*}\right)\right]\left(r^{\prime \prime}\right) d r^{\prime \prime}-k \alpha\left(r^{\prime}\right) \int_{\Omega} \alpha^{\prime}\left(r^{\prime \prime}\right)\left[x_{1}(t)\right]\left(r^{\prime \prime}\right) d r^{\prime \prime}\right)\right] d r^{\prime} d r .
\end{aligned}
$$

Using successively (15), the fact that $a b \leq\left(a^{2}+b^{2}\right) / 2$ for all $a, b \in \mathbb{R}$, and Cauchy-Schwarz inequality, it can be seen that

$$
\begin{aligned}
\dot{W}^{(23 a)} \leq & -\frac{2}{\tau_{1}^{*}} W+\ell_{1} \int_{\Omega} \int_{\Omega}\left|\left[x_{1}(t)\right](r)-\left[x_{1}(t)\right]\left(r^{\prime}\right)\right| \\
& \times\left|\int_{\Omega} \sum_{i=1}^{2}\left(w_{1 i}\left(r, r^{\prime \prime}\right)-w_{1 i}\left(r^{\prime}, r^{\prime \prime}\right)\right)\left[x_{i}\left(t-d_{i}^{*}\right)\right]\left(r^{\prime \prime}\right) d r^{\prime \prime}-k\left(\alpha(r)-\alpha\left(r^{\prime}\right)\right) \int_{\Omega} \alpha^{\prime}\left(r^{\prime \prime}\right)\left[x_{1}(t)\right]\left(r^{\prime \prime}\right) d r^{\prime \prime}\right| d r^{\prime} d r \\
\leq & -\frac{1}{\tau_{1}^{*}} W+\frac{\ell_{1}^{2}}{2} \int_{\Omega} \int_{\Omega}\left(\int_{\Omega} \sum_{i=1}^{2}\left(w_{1 i}\left(r, r^{\prime \prime}\right)-w_{1 i}\left(r^{\prime}, r^{\prime \prime}\right)\right)\left[x_{i}\left(t-d_{i}^{*}\right)\right]\left(r^{\prime \prime}\right) d r^{\prime \prime}\right. \\
& \left.-k\left(\alpha(r)-\alpha\left(r^{\prime}\right)\right) \int_{\Omega} \alpha^{\prime}\left(r^{\prime \prime}\right)\left[x_{1}(t)\right]\left(r^{\prime \prime}\right) d r^{\prime \prime}\right)^{2} d r^{\prime} d r \\
\leq & -\frac{1}{\tau_{1}} W+\ell_{1}^{2} \int_{\Omega} \int_{\Omega}\left(\int_{\Omega} \sum_{i=1}^{2}\left(w_{1 i}\left(r, r^{\prime \prime}\right)-w_{1 i}\left(r^{\prime}, r^{\prime \prime}\right)\right)\left[x_{i}\left(t-d_{i}^{*}\right)\right]\left(r^{\prime \prime}\right) d r^{\prime \prime}\right)^{2} d r^{\prime} d r \\
& +k \ell_{1}^{2} \int_{\Omega} \int_{\Omega}\left(\left(\alpha(r)-\alpha\left(r^{\prime}\right)\right) \int_{\Omega} \alpha^{\prime}\left(r^{\prime \prime}\right)\left[x_{1}(t)\right]\left(r^{\prime \prime}\right) d r^{\prime \prime}\right)^{2} d r^{\prime} d r \\
\leq & -\frac{1}{\tau_{1}^{*}} W+2 \ell_{1}^{2} \int_{\Omega} \int_{\Omega}\left(\sum_{i=1}^{2} \int_{\Omega}\left(w_{1 i}\left(r, r^{\prime \prime}\right)-w_{1 i}\left(r^{\prime}, r^{\prime \prime}\right)\right)^{2} d r^{\prime \prime} \int_{\Omega}\left[x_{i}\left(t-d_{i}^{*}\right)\right]\left(r^{\prime \prime}\right)^{2} d r^{\prime \prime}\right) d r^{\prime} d r \\
& +k \ell_{1}^{2} \int_{\Omega} \int_{\Omega}\left(\left(\alpha(r)-\alpha\left(r^{\prime}\right)\right)^{2} \int_{\Omega} \alpha^{\prime}\left(r^{\prime \prime}\right)^{2} d r^{\prime \prime} \int_{\Omega}\left[x_{1}(t)\right]\left(r^{\prime \prime}\right)^{2} d r^{\prime \prime}\right) d r^{\prime} d r .
\end{aligned}
$$

Furthermore, since the activation functions $S_{i}$ are bounded, for all initial conditions lying in their image, the solutions remain in it. More precisely, for all $x_{i 0} \in C\left(\left[-d_{i}^{*} ; 0\right],\left(\underline{S}_{i} ; \bar{S}_{i}\right)\right)$, it holds that $\underline{S}_{i} \leq\left[x_{i}(t)\right](r) \leq \bar{S}_{i}$ for all $t \geq 0$ and all $r \in \Omega$. In particular, it holds that $\left|\left[x_{i}(t)\right](r)\right| \leq \overline{\bar{S}}$ for all $t \geq-d_{i}^{*}$ and all $r \in \Omega$. It follows that

$$
\begin{aligned}
\dot{W}^{(23 a)} \leq & -\frac{1}{\tau_{1}^{*}} W+2 \ell_{1}^{2} \overline{\bar{S}}^{2} \#_{\Omega} \int_{\Omega} \int_{\Omega} \int_{\Omega} \sum_{i=1}^{2}\left(w_{1 i}\left(r, r^{\prime \prime}\right)-w_{1 i}\left(r^{\prime}, r^{\prime \prime}\right)\right)^{2} d r^{\prime \prime} d r^{\prime} d r \\
& +k \ell_{1}^{2} \overline{\bar{S}}^{2} \#_{\Omega} \int_{\Omega} \int_{\Omega}\left(\left(\alpha(r)-\alpha\left(r^{\prime}\right)\right)^{2} \int_{\Omega} \alpha^{\prime}\left(r^{\prime \prime}\right)^{2} d r^{\prime \prime}\right) d r^{\prime} d r .
\end{aligned}
$$

Using the notation of (24)-(25) and letting $c:=\ell_{1}^{2} \overline{\bar{S}}^{2} \#_{\Omega}$, we obtain $\dot{W}^{(23 a)} \leq-\frac{1}{\tau_{1}^{*}} W+2 c \sum_{i=1}^{2} \mathcal{H}\left(w_{1 i}\right)^{2}+k c\left\|^{\prime}\right\|_{\mathcal{F}}^{2} \mathcal{H}(\alpha)^{2}$. The conclusion follows by integrating and replacing $W$ by its expression.

Claim 2 Letting $\bar{\alpha}:=\max _{r \in \Omega} \alpha(r)$, it holds that, for all $t \geq 0$ and all $r \in \Omega$,

$$
\int_{\Omega}\left(\int_{\Omega} \alpha^{\prime}\left(r^{\prime}\right)\left[x_{1}(t)\right]\left(r^{\prime}\right) d r^{\prime}-\left[x_{1}(t)\right](r)\right)^{2} d r \leq \bar{\alpha}^{2}\left\|\alpha^{\prime}\right\|_{\mathcal{F}}^{2} \mathcal{H}\left(x_{1}(t)\right)^{2}
$$


Proof For each $t \geq 0$ and each $r, r^{\prime} \in \Omega$, let $\xi\left(r, r^{\prime}, t\right):=\left[x_{1}(t)\right]\left(r^{\prime}\right)-\left[x_{1}(t)\right](r)$. Recalling that $\int_{\Omega} \alpha^{\prime}(r) d r=1$,

$$
\begin{aligned}
\int_{\Omega}\left(\alpha(r) \int_{\Omega} \alpha^{\prime}\left(r^{\prime}\right)\left[x_{1}(t)\right]\left(r^{\prime}\right) d r^{\prime}-\left[x_{1}(t)\right](r)\right)^{2} d r & =\int_{\Omega} \alpha(r)^{2}\left(\int_{\Omega} \alpha^{\prime}\left(r^{\prime}\right)\left(\left[x_{1}(t)\right](r)+\xi\left(r, r^{\prime}, t\right)\right) d r^{\prime}-\left[x_{1}(t)\right](r)\right)^{2} d r \\
& \leq \int_{\Omega} \alpha(r)^{2}\left(\left[x_{1}(t)\right](r) \int_{\Omega} \alpha^{\prime}\left(r^{\prime}\right) d r^{\prime}+\int_{\Omega} \alpha^{\prime}\left(r^{\prime}\right) \xi\left(r, r^{\prime}, t\right) d r^{\prime}-\left[x_{1}(t)\right](r)\right)^{2} d r \\
& \leq \int_{\Omega} \alpha(r)^{2}\left(\int_{\Omega} \alpha^{\prime}\left(r^{\prime}\right) \xi\left(r, r^{\prime}, t\right) d r^{\prime}\right)^{2} d r \\
& \leq\left\|\alpha^{\prime}\right\|_{\mathcal{F}}^{2} \int_{\Omega} \alpha(r)^{2} \int_{\Omega} \xi\left(r, r^{\prime}, t\right)^{2} d r^{\prime} d r \\
& \leq\left\|\alpha^{\prime}\right\|_{\mathcal{F}}^{2} \bar{\alpha}^{2} \int_{\Omega} \int_{\Omega} \xi\left(r, r^{\prime}, t\right)^{2} d r^{\prime} d r .
\end{aligned}
$$

The conclusion follows by observing that, in view of $(24), \int_{\Omega} \int_{\Omega} \xi\left(r, r^{\prime}, t\right)^{2} d r^{\prime} d r=\mathcal{H}\left(x_{1}(t)\right)^{2}$.

Based on these two claims, we are now ready to complete the proof of Proposition 4. Recalling that the delay distributions $d_{1}$ and $d_{2}$ are uniform, the coupled neural fields (23) studied here can be written as

$$
\begin{aligned}
& \tau_{1}(r)\left[\dot{x}_{1}(t)\right](r)=S_{1}\left(r, \int_{\Omega} \sum_{i=1}^{2} w_{1 i}\left(r, r^{\prime}\right)\left[x_{i}\left(t-d_{1}^{*}\right)\right]\left(r^{\prime}\right) d r^{\prime}-k \alpha(r)\left[x_{1}(t)\right](r)+\left[p_{1}(t)\right](r)\right)-\left[x_{1}(t)\right](r) \\
& \tau_{2}(r)\left[\dot{x}_{2}(t)\right](r)=-\left[x_{2}(t)\right](r)+S_{2}\left(r, \int_{\Omega} \sum_{i=1}^{2} w_{2 i}\left(r, r^{\prime}\right)\left[x_{i}\left(t-d_{2}^{*}\right)\right]\left(r^{\prime}\right) d r^{\prime}\right)
\end{aligned}
$$

where $\left[p_{1}(t)\right](r)=-k \alpha(r) \int_{\Omega} \alpha^{\prime}\left(r^{\prime}\right)\left[x_{1}(t)\right]\left(r^{\prime}\right) d r^{\prime}+k \alpha(r)\left[x_{1}(t)\right](r)$. Since $k \geq k^{*}$, we know from Proposition 3 that this system is ISS. In particular, due to the asymptotic gain property induced by ISS (see (7)), there exists $\nu \in \mathcal{K}_{\infty}$ such that

$$
\limsup _{t \rightarrow \infty}\|x(t)\|_{\mathcal{F}} \leq \nu\left(\limsup _{t \rightarrow \infty}\left\|p_{1}(t)\right\|_{\mathcal{F}}\right) .
$$

Furthermore, using Claim 2, $\left\|p_{1}(t)\right\|_{\mathcal{F}}^{2} \leq k^{2} \bar{\alpha}^{2}\left\|\alpha^{\prime}\right\|_{\mathcal{F}}^{2} \mathcal{H}\left(x_{1}(t)\right)^{2}$. Consequently, Claim 1 gives that

$$
\limsup _{t \rightarrow \infty}\left\|p_{1}(t)\right\|_{\mathcal{F}} \leq k \bar{\alpha}\left\|\alpha^{\prime}\right\|_{\mathcal{F}} \ell_{1} \overline{\bar{S}} \sqrt{\frac{2 \# \Omega}{\tau_{1}^{*}}} \sqrt{2 \sum_{i=1}^{2} \mathcal{H}\left(w_{1 i}\right)^{2}+k\left\|\alpha^{\prime}\right\|_{\mathcal{F}}^{2} \mathcal{H}(\alpha)^{2}} .
$$

It thus follows from (47) that

$$
\begin{aligned}
\limsup _{t \rightarrow \infty}\|x(t)\|_{\mathcal{F}} & \leq \nu\left(k \bar{\alpha}\left\|\alpha^{\prime}\right\|_{\mathcal{F}} \ell_{1} \overline{\bar{S}} \sqrt{\frac{2 \# \Omega}{\tau_{1}^{*}}} \sqrt{2 \sum_{i=1}^{2} \mathcal{H}\left(w_{1 i}\right)^{2}+k\left\|\alpha^{\prime}\right\|_{\mathcal{F}}^{2} \mathcal{H}(\alpha)^{2}}\right) \\
& \leq \nu\left(k c \sqrt{2 \sum_{i=1}^{2} \mathcal{H}\left(w_{1 i}\right)^{2}}+k^{3 / 2} c\left\|\alpha^{\prime}\right\|_{\mathcal{F}} \mathcal{H}(\alpha)\right) \\
& \leq \nu\left(2 k c \sqrt{2 \sum_{i=1}^{2} \mathcal{H}\left(w_{1 i}\right)^{2}}\right)+\nu\left(2 k^{3 / 2} c\left\|^{\prime}\right\|_{\mathcal{F}} \mathcal{H}(\alpha)\right) \\
& \leq \nu\left(4 k c \sum_{i=1}^{2} \mathcal{H}\left(w_{1 i}\right)\right)+\nu\left(2 k^{3 / 2} c\left\|^{\prime}\right\|_{\mathcal{F}} \mathcal{H}(\alpha)\right)
\end{aligned}
$$

where $c:=\bar{\alpha}\left\|\alpha^{\prime}\right\|_{\mathcal{F}} \ell_{1} \overline{\bar{S}} \sqrt{\frac{2 \#_{\Omega}}{\tau_{1}^{*}}}$. The conclusion follows by picking $\nu_{1}(s):=\nu(4 k c s)$ and $\nu_{2}(s):=\nu\left(2 k^{3 / 2} c\left\|\alpha^{\prime}\right\| s\right)$ for all $s \geq 0$. 


\subsection{Proof of Proposition 5}

Omitting some arguments for the sake of clarity, the coupled neural fields (27) studied here can be written as

$$
\begin{aligned}
& \tau_{1}(r)\left[\dot{x}_{1}(t)\right](r)=-\left[x_{1}(t)\right](r)+S_{1}\left(r,\left[p_{1}(t)\right](r)+\int_{\Omega} \sum_{i=1}^{2} w_{1 i}\left(r, r^{\prime}\right)\left[x_{i}\left(t-d_{i}\right)\right]\left(r^{\prime}\right) d r^{\prime}-k \alpha(r)\left[x_{1}(t)\right](r)\right) \\
& \tau_{2}(r)\left[\dot{x}_{2}(t)\right](r)=-\left[x_{2}(t)\right](r)+S_{2}\left(r, \int_{\Omega} \sum_{i=1}^{2} w_{2 i}\left(r, r^{\prime}\right)\left[x_{i}\left(t-d_{i}\right)\right]\left(r^{\prime}\right) d r^{\prime}\right),
\end{aligned}
$$

where $\left.\left[p_{1}(t)\right](r)=-k \alpha(r)\left(\left[x_{1}\left(t-d_{c}(t, r)\right)\right](r)-x_{1}(t)\right](r)\right)$. Since $k \geq k^{*}$, we know from Proposition 3 that this system is ISS. The asymptotic gain property (7) then ensures the existence of $\tilde{\nu} \in \mathcal{K}_{\infty}$ such that

$$
\limsup _{t \rightarrow \infty}\|x(t)\|_{\mathcal{F}} \leq \tilde{\nu}\left(\limsup _{t \rightarrow \infty}\left\|p_{1}(t)\right\|_{\mathcal{F}}\right) .
$$

Now, let $\overline{\bar{S}}_{1}:=\max \left\{\left|\underline{S}_{1}\right| ;\left|\bar{S}_{1}\right|\right\}$. Then as stressed in the proof of Proposition 4 , for all $x_{10} \in C\left([-\bar{d} ; 0],\left(\underline{S}_{1} ; \bar{S}_{1}\right)\right)$, it holds that $\underline{S}_{1} \leq\left[x_{1}(t)\right](r) \leq \bar{S}_{1}$ for all $t \geq 0$ and all $r \in \Omega$. In particular, it holds that $\left|\left[x_{1}(t)\right](r)\right| \leq \overline{\bar{S}}_{1}$ for all $t \geq-\bar{d}$ and all $r \in \Omega$. In view of $(27 \mathrm{a})$, it follows that $\left|\left[\dot{x}_{1}(t)\right](r)\right| \leq 2 \overline{\bar{S}}_{1} / \tau_{1}(r)$. Consequently, $\left|\left[x_{1}(t)\right](r)-\left[x_{1}\left(t-d_{c}(t, r)\right)\right](r)\right| \leq$ $2 \overline{\bar{S}}_{1} \bar{d}_{c} / \tau_{1}(r)$, where $\bar{d}_{c}:=\sup _{t \geq 0, r \in \Omega} d_{c}(t, r)$. It follows from $(48)$ that $\limsup _{t \rightarrow \infty}\|x(t)\| \mathcal{F} \leq \tilde{\nu}\left(2 k\left\|_{\alpha}\right\|_{\mathcal{F}} \overline{\bar{S}}_{1} \bar{d}_{c}\left\|1 / \tau_{1}\right\|_{\mathcal{F}}\right)$. Letting $\nu(s):=\tilde{\nu}\left(2 k\|\alpha\|_{\mathcal{F}} \overline{\bar{S}}_{1}\left\|1 / \tau_{1}\right\|_{\mathcal{F}} s\right)$ for all $s \geq 0$, the conclusion follows.

\section{References}

Amari, S.-I., 1977. Dynamics of pattern formation in lateral-inhibition type neural fields. Biological Cybernetics $27(2), 77-87$.

Atay, F., Hutt, A., 2006. Neural fields with distributed transmission speeds and long-range feedback delays. SIAM Journal on Applied Dynamical Systems 5 (4).

Atay, F. M., Hutt, A., 2004. Stability and Bifurcations in Neural Fields with Finite Propagation Speed and General Connectivity. SIAM Journal on Applied Mathematics 65 (2), 644-666.

Batista, C., Lopes, S., Viana, R., Batista, A., 2010. Delayed feedback control of bursting synchronization in a scale-free neuronal network. Neural Networks 23 (1), 114-124.

beim Graben, P., Hutt, A., 2014. Attractor and saddle node dynamics in heterogeneous neural fields. EPJ Nonlinear biomedical physics 2 (4), 1-17.

Benabid, A., Pollak, P., Gervason, C., Hoffmann, D., Gao, D., Hommel, M., Perret, J., de Rougemont, J., 1991. Long-term suppression of tremor by chronic stimulation of the ventral intermediate thalamic nucleus. The Lancet 337, 403-406.

Bressloff, P., 2012. Spatiotemporal dynamics of continuum neural fields. Journal of Physics A: Mathematical and Theoretical 45 (3), 033001.

Butson, C. R., McIntyre, C. C., 2005. Tissue and electrode capacitance reduce neural activation volumes during deep brain stimulation. Clinical neurophysiology 116 (10), 2490-2500.

Carron, R., Chaillet, A., Filipchuk, A., Pasillas-Lépine, W., Hammond, C., 2013. Closing the Loop of Deep Brain Stimulation. Frontiers in Systems Neuroscience 13 (112), 1-18.

Coombes, S., beim Graben, P., Potthast, R., Wright, J., 2014. Neural Fields: Theory and Applications. Springer.

Dashkovskiy, S., Mironchenko, A., 2013. Input-to-state stability of infinite-dimensional control systems. Math. of Control, Signals, and Systems 25 (1), 1-35.

Dashkovskiy, S., Rueffer, B., Wirth, F., 2010. Small gain theorems for large scale systems and construction of ISS Lyapunov functions. SIAM Journal on Control and Optimization 48 (6), 4089-4118.

Detorakis, G., Chaillet, A., Palfi, S., Senova, S., 2015. Closed-loop stimulation of a delayed neural fields model of parkinsonian STN-GPe network: a theoretical and computational study. Frontiers in Neuroscience 9 (237).

Faugeras, O., Veltz, R., Grimbert, F., 2009. Persistent neural states: stationary localized activity patterns in nonlinear continuous n-population, q-dimensional neural networks. Neural Computation 21 (1), 147-187.

Faye, G., Faugeras, O., 2010. Some theoretical and numerical results for delayed neural field equations. Physica D: Nonlinear Phenomena 239 (9), 561-578. 
Feng, X., Greenwald, B., Rabitz, H., Shea-Brown, E., Kosut, R., 2007. Toward closed-loop optimization of deep brain stimulation for Parkinson's disease: concepts and lessons from a computational model. Journal of neural engineering 4 (2), L14.

Gradinaru, V., Mogri, M., Thompson, K., Henderson, J., Deisseroth, K., 2009. Optical deconstruction of parkinsonian neural circuitry. Science 324 (5925), 354-359.

Grant, P., Lowery, M. M., 2013. Simulation of cortico-basal ganglia oscillations and their suppression by closed loop deep brain stimulation. IEEE Transactions on neural systems and rehabilitation engineering 21 (4), 584-594.

Graupe, D., Basu, I., Tuninetti, D., Vannemreddy, P., Slavin, K., 2010. Adaptively controlling deep brain stimulation in essential tremor patient via surface electromyography. Neurological research 32 (9), 899-904.

Grossman, N., Nikolic, K., Toumazou, C., Degenaar, P., 2011. Modeling Study of the Light Stimulation of a Neuron Cell With Channelrhodopsin-2 Mutants. IEEE Trans. on Biomedical Engineering 58 (6), 1742-1751.

Haidar, I., Pasillas-Lépine, W., Chaillet, A., Panteley, E., Palfi, S., Senova, S., 2016. A firing-rate regulation strategy for closed-loop deep brain stimulation. Biological Cybernetics 110 (1), 55-71.

Jacques, S. L., 2013. Optical properties of biological tissues: a review. Physics in medicine and biology 58 (11), R37.

Jiang, Z., Mareels, I., Wang, Y., 1996. A Lyapunov formulation of nonlinear small gain theorem for interconnected systems. Automatica 32 (8), 1211-1215.

Karafyllis, I., Jiang, Z.-P., 2007. A small-gain theorem for a wide class of feedback systems with control applications. SIAM Journal on Control and Optimization 46 (4), 1483-1517.

Karafyllis, I., Jiang, Z. P., 2011. A vector small-gain theorem for general non-linear control systems. IMA J. Math. Control \& Information 28 (3), 309-344.

Karafyllis, I., Pepe, P., Jiang, Z., 2008. Global output stability for systems described by retarded functional differential equations: Lyapunov characterizations. European Journal of Control 14 (6), 516-536.

Laing, C. R., Troy, W. C., Gutkin, B., Ermentrout, G., 2002. Multiple bumps in a neuronal model of working memory. SIAM Journal on Applied Mathematics 63 (1), 62-97.

Little, S., Beudel, M., Zrinzo, L., Foltynie, T., Limousin, P., Hariz, M., Neal, S., Cheeran, B., Cagnan, H., Gratwicke, J., Aziz, T. Z., Pogosyan, A., Brown, P., 2016. Bilateral adaptive deep brain stimulation is effective in Parkinson's disease. Journal of Neurology, Neurosurgery \& Psychiatry 87, 717-721.

Lysyansky, B., Popovych, O., Tass, P., 2011. Desynchronizing anti-resonance effect of m:n ON-OFF coordinated reset stimulation. Journal of Neural Engineering 8, 036019.

Marceglia, S., Rossi, L., Foffani, G., Bianchi, A., Cerutti, S., Priori, A., 2007. Basal ganglia local field potentials: applications in the development of new deep brain stimulation devices for movement disorders. Expert review of medical devices 4 (5), 605-614.

Mazenc, F., Malisoff, M., Lin, Z., 2008. Further results on input-to-state stability for nonlinear systems with delayed feedbacks. Automatica 44 (9), 2415-2421.

Mironchenko, A., 2012. Input-to-state stability of infinite-dimensional control systems, PhD thesis (University of Bremen).

Mironchenko, A., Ito, H., Sep. 2016. Characterizations of integral input-to-state stability for bilinear systems in infinite dimensions. Mathematical Control and Related Fields 6 (3), 447-466.

Nevado-Holgado, A., Terry, J., Bogacz, R., 2010. Conditions for the generation of beta oscillations in the subthalamic nucleus-globus pallidus network. Journal of Neuroscience 30 (37), 12340-12352.

Pasillas-Lépine, W., 2013. Delay-induced oscillations in Wilson and Cowan's model: An analysis of the subthalamopallidal feedback loop in healthy and parkinsonian subjects. Biological Cybernetics 107 (3), 289-308.

Pepe, P., 2007. The problem of the absolute continuity for Lyapunov-Krasovskii functionals. IEEE Trans. Autom. Control 52 (5), 953-957.

Pepe, P., Jiang, Z. P., 2006. A Lyapunov-Krasovskii methodology for ISS and iISS of time-delay systems. Systems \& Control Letters 55 (12), 1006-1014.

Pfister, J.-P., Tass, P. A., 2010. STDP in oscillatory recurrent networks: theoretical conditions for desynchronization and applications to deep brain stimulation. Frontiers in computational neuroscience 4 (22), 374-383.

Pinto, D. J., Ermentrout, G., 2001. Spatially structured activity in synaptically coupled neuronal networks: I. Traveling fronts and pulses. SIAM Journal on Applied Mathematics 62 (1), 206-225.

Plenz, D., Kital, S., 1999. A basal ganglia pacemaker formed by the subthalamic nucleus and external globus pallidus. Nature 400, 677-682.

Prieur, C., Mazenc, F., 2012. ISS-Lyapunov functions for time-varying hyperbolic systems of balance laws. Mathematics of Control, Signals, and Systems 24 (1), 111-134.

Reutsky-Gefen, I., Golan, L., Farah, N., Schejter, A., Tsur, L., Brosh, I., Shoham, S., 2013. Holographic optogenetic stimulation of patterned neuronal activity for vision restoration. Nature Communications 4 (1509).

Rosin, B., Slovik, M., Mitelman, R., Rivlin-Etzion, M., Haber, S., Israel, Z., Vaadia, E., Bergman, H., 2011. Closedloop deep brain stimulation is superior in ameliorating Parkinsonism. Neuron 72 (2), 370-384.

Santaniello, S., Fiengo, G., Glielmo, L., Grill, W., 2011. Closed-loop control of deep brain stimulation: A simulation 
study. IEEE Trans. on Neural Systems and Rehabilitation Engineering 19 (1), 15-24.

Sontag, E., 1989. Smooth stabilization implies coprime factorization. IEEE Trans. Autom. Control 34 (4), 435-443.

Sontag, E., 2008. Input to state stability: Basic concepts and results. Lecture Notes in Mathematics. Springer-Verlag, Berlin, pp. 163-220.

Tass, P. A., Qin, L., Hauptmann, C., Dovero, S., Bezard, E., Boraud, T., Meissner, W. G., 2012. Coordinated reset has sustained aftereffects in Parkinsonian monkeys. Ann. Neurol. 72 (5), 816-820.

Teel, A., 1998. Connections between Razumikhin-type theorems and the ISS nonlinear small gain theorem. IEEE Trans. Autom. Control 43 (7), 960-964.

Veltz, R., Faugeras, O., 2010. Local/global analysis of the stationary solutions of some neural field equations. SIAM Journal on Applied Dynamical Systems 9 (3), 954-998.

Veltz, R., Faugeras, O., 2011. Stability of the stationary solutions of neural field equations with propagation delays. The Journal of Mathematical Neuroscience 1 (1), 1-28.

Wagenaar, D., Madhavan, R., Pine, J., Potter, S., 2005. Controlling bursting in cortical cultures with closed-loop multi-electrode stimulation. The Journal of neuroscience 25 (3), 680-688.

Wilson, H., Cowan, J., 1973. A mathematical theory of the functional dynamics of cortical and thalamic nervous tissue. Kybernetic 13, 55-80. 